\title{
Gradhiva
}

Revue d'anthropologie et d'histoire des arts

$14 \mid 2011$

Carl Einstein et les primitivismes

\section{Apocalypse primitive}

Une lecture politique de Negerplastik

Primitive Apocalypse. A political reading of Negerplastik

\section{Maria Stavrinaki}

\section{OpenEdition}

Journals

Édition électronique

URL : http://journals.openedition.org/gradhiva/2287

DOI : 10.4000/gradhiva.2287

ISSN : 1760-849X

Éditeur

Musée du quai Branly Jacques Chirac

Édition imprimée

Date de publication : 30 novembre 2011

Pagination : $56-77$

ISBN : 978-2-35744-046-3

ISSN : 0764-8928

Référence électronique

Maria Stavrinaki, «Apocalypse primitive », Gradhiva [En ligne], 14 | 2011, mis en ligne le 30 novembre 2014, consulté le 01 mai 2019. URL : http://journals.openedition.org/gradhiva/2287 ; DOI : 10.4000/ gradhiva.2287

(c) musée du quai Branly 


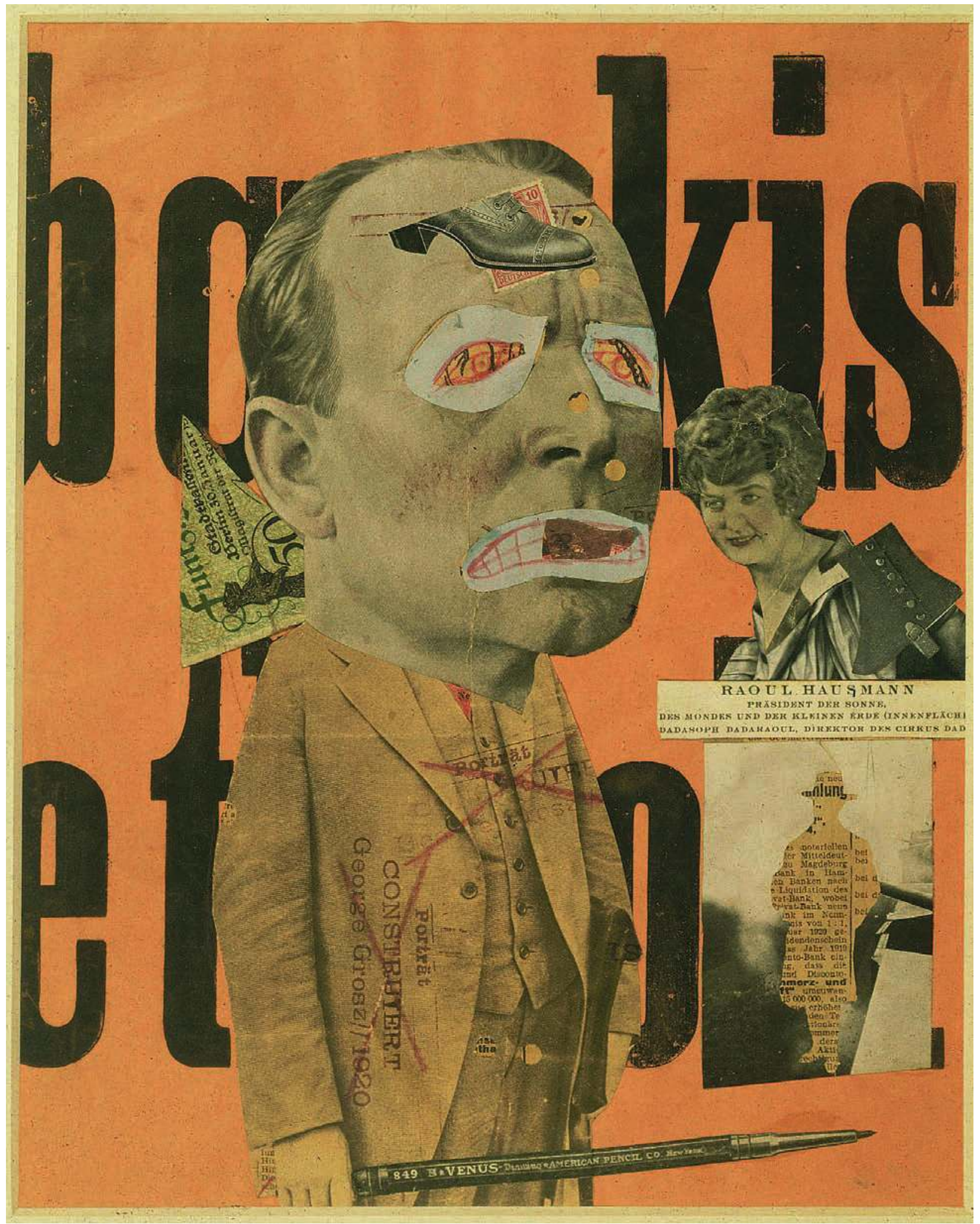

Fig. 1 Raoul Hausmann, Der Kunstkritiker, 1919-1920 @ BPK, Berlin, dist. RMN / Hermann Buresch. 


\section{Apocalypse primitive}

\section{Une lecture politique de Negerplastik}

Maria Stavrinaki

-

1. Ernst Bloch, L'Esprit de l'utopie, [1918/1923], trad. Anne-Marie Lang et Catherine Piron-Audard, Paris, Gallimard, 1989, p. 58-60.
Carl Einstein était hanté par l'impératif de la rupture du continuum de l'histoire par la révolte subjective et par son amplification espérée en révolution collective. Le plus souvent critique à l'égard du marxisme orthodoxe, il a cherché dans les ressources «nomades » de la subjectivité - ces capacités fictionnantes qui ne se limitent pas à ce qu'Ernst Bloch appelait «le présent sociologique ${ }^{1}$ - le supplément qui pourrait arracher la révolution à son assise exclusivement matérialiste : imagination, disposition à l'hallucination, inconscient ou mémoire involontaire d'un passé ancestral se sont chargés, à différents moments de sa vie, de cette tâche. Méfiant à l'égard de toute conception automatique du temps historique, supposé s'autoproduire au rythme de lois qui lui seraient immanentes, Einstein condamnait la contamination de l'histoire par les sciences naturelles sous la forme du déterminisme marxiste et de l'évolutionnisme cher à la social-démocratie de son temps. Cet homme était autrement pressé que ses contemporains adeptes du progrès : sa critique de l'attentisme socialiste, dont il s'appliquait à déconstruire régulièrement le postulat optimiste, allait de pair avec son désir de précipiter le temps, de le brusquer jusqu'à le briser. Marqué par Georges Sorel, le penseur du syndicalisme révolutionnaire qui a compté plus que quiconque dans la révision du marxisme de droite et de gauche, il estimait que la "catastrophe " révolutionnaire préconisée par Karl Marx ne se donnait pas les moyens de son avènement. C'est sur le postulat d'une révolution d'abord subjective que les deux axes principaux de la pensée d'Einstein, l'art et la politique, ont pu nouer une relation puissante, mais conflictuelle parce que soumise aux contradictions qui lui étaient propres et aux variations tumultueuses de l'histoire.

C'est un fait : Einstein a pu attribuer à l'art de son temps un pouvoir inouï - mettre fin à l'automatisme de l'histoire en façonnant à travers l'organisation du sensible une autre ontologie politique pour le sujet moderne. La fin de l'imitation dans l'art avait donc selon lui partie liée avec la fin de l'histoire «imitative», se 


\section{CARL EINSTEIN ET LES PRIMITIVISMES}

- -

2. Terme employé dans son texte «Anmerkungen » (publié dans Die Aktion, 1912), Werke, t. I (19071918), Hermann Haarmann et Klaus Siebenhaar (éd.), Berlin, Fannei \& Walz, 1994, p. 142-143.

3. Carl Einstein, «Bemerkungen zum heutigen Kunstbetrieb », Werke, t. I, op. cit., p. 140. Une autre interprétation, tout à fait positive, du cubisme en général et de Picasso en particulier, se relève pour la première fois dans son texte «Herbstausstellung am Kurfürstendamm » (publié dans Die Aktion, 1913), Werke, t. I, op. cit., p. 170-173.

4. Carl Einstein, «Anmerkungen », art. cit. Je remercie Charles Haxthausen d'avoir contribué à dissiper mes doutes sur ce texte.

5. Ibid., p. 142. réalisant comme à l'insu de ses contemporains. Il inscrivait ainsi l'art et la révolution modernes dans une généalogie inédite, qu'il faisait remonter à la sculpture africaine. Transposée dans le contexte moderne et ainsi détournée de ses historicité et finalité propres, la sculpture africaine lui semblait pouvoir procurer par la forme et la temporalité qu'elle imposait au spectateur la preuve immédiatement visible, et par-là même ô combien précieuse, que l'art était capable d'agir de manière «catastrophique » [katastrophal $\left.{ }^{2}\right]$.

\section{Comme une catastrophe}

En 1912 encore, l'art de son temps ne semblait pas suffisant à Einstein pour changer la réalité politique. Il suivait attentivement les expériences formelles des artistes modernes, mais restait sceptique vis-à-vis de leurs prétentions métaphysiques - réelles ou supposées. Se heurtant à l'aporie constitutive d'un art subjectif à visée objective, il relevait, d'un côté, le langage «tectonique " de Pablo Picasso, mais constatait, de l'autre, l'absence d'architecture, art synthétique par excellence auquel ce langage pictural aurait pu s'intégrer. Il trouvait que, faute de fondement métaphysique et de religion, Picasso succombait fatalement à des contradictions formelles ${ }^{3}$. En somme, le cubisme ne répondait pas encore aux exigences organicistes d'Einstein. Mais du côté de la politique, ce dernier prenait également ses distances vis-à-vis d'un certain expressionnisme activiste qui, comme lui-même, en appelait aux ressources de la subjectivité pour renverser l'ordre existant entretenu par la social-démocratie et, plus généralement, par la démocratie parlementaire. Son article "Anmerkungen» ("Notes»; 1912), publié comme tous ses autres textes politiques de ces années-là dans la revue expressionniste de gauche Die Aktion, est un texte qui peut paraître de prime abord sibyllin $^{4}$. Mais si on le lit de manière transversale et non pas autonome, comme le premier d'un ensemble de textes politiques écrits entre 1912 et 1914, on réalise que l'enjeu d'“Anmerkungen " était de montrer ce qu'était, selon Einstein, une révolte nihiliste, c'est-à-dire une révolte ne pouvant aboutir à rien, si ce n'est à la légitimation de l'ordre existant.

Einstein décrivait dans ce texte l'intellectuel qui avait fait de la révolte un métier et qu'il appelait, non sans ironie, le « révolteur ». Au discours de ce dernier, il reconnaissait d'entrée de jeu des qualités qu'il valorisait éminemment : ainsi ce discours était-il "catastrophique", "primitif», "non dialectique». La révolte avait une intransigeance qui contrastait sévèrement avec les pratiques graduelles et feutrées du libéralisme politique. La dialectique, cette méthode logique qu'il trouvait objectivée dans les assemblées modernes, lui semblait un "équilibre des oppositions, [une] légitimation esthétique ou historique ». Purement formelle, la démocratie libérale apparaissait donc à Einstein comme une neutralisation judicieuse des conflits. Et s'il mesurait cette efficacité politique de la démocratie sur le plan esthétique et sur celui de l'histoire, c'était parce que tous ces moments étaient parfaitement liés dans sa pensée. Pour tout dire, les notions importantes de l'ontologie einsteinienne étaient liées par une réciprocité métaphorique, les courants esthétiques se traduisant en régimes politiques, les régimes politiques en régimes temporels, etc. Que la contre-révolution en art ait été menée par la mimèsis, dont le dernier rejeton aurait été l'impressionnisme, voilà une thèse bien entendu largement répandue dans le discours moderniste de l'époque; mais, selon Einstein, il y avait aussi une sorte de mimèsis politique : 


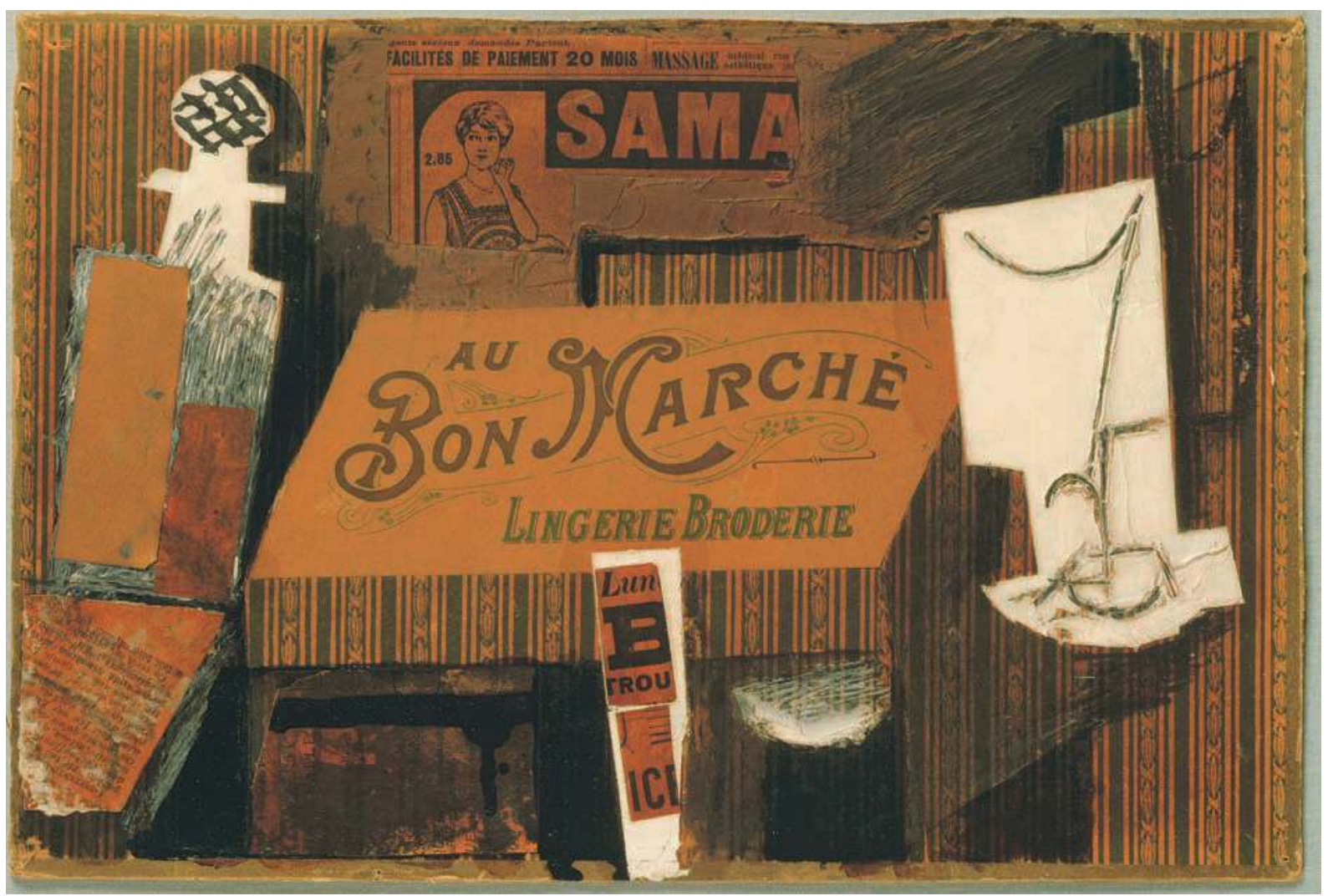

Fig. 2 Pablo Picasso, Au Bon Marché, 1913, Peter et Irene Ludwig Stiftung, prêté au Ludwig Museum, Cologne @ Succession Picasso 2011.

celle qu'entretenait, par exemple, la démocratie parlementaire. La dialectique, par sa visée neutralisante, agissait finalement comme une "belle apparence", capable d'estomper les rapports contrastés générateurs du changement. La révolte, en revanche, était « primitive » dans sa forme. Cette primitivité signifiait une attitude directe, indépendante de toute médiation : à la différence de la représentation démocratique, la révolte pouvait faire mal et elle pouvait s'incarner en un nombre de sujets illimité. Enfin, la médiation et la dialectique démocratiques se traduisaient dans cette conception graduelle du temps historique qu'était l'évolutionnisme, tandis que l'immédiateté primitive de la révolte se traduisait en désir d'une «catastrophe » ayant lieu ici et maintenant.

Au demeurant, si Einstein abordait dans son texte la "révolte" en tant que discours plutôt qu'en tant que praxis, cela était dû au fait que, selon lui, elle en était intrinsèquement incapable. Son fanatisme restait idéel, métaphysique, parfaitement désincarné. "Non dogmatique», parce que n'aspirant pas à se verser dans le réel par peur d'y sacrifier son intégrité, la révolte restait encore et toujours une Idée. Flottant dans la sphère métaphysique et renonçant à forger une « loi », le «révolteur » était finalement l'homme qui fuyait l'histoire : "Parce que pour lui l'histoire est totalement insignifiante, et même fausse en soi, l'idée n'ayant pu s'y réaliser jamais ${ }^{6}$. " Voilà pourquoi ce fanatique de l'esprit était, aux yeux d'Einstein, sinon un réactionnaire, du moins un légitimiste. Il permettait à l'histoire de reprendre tranquillement son cours, comme une répétition, un infernal retour du même.

-

6. Ibid., p. 143. 


\section{${ }_{4}^{20}$ DER $\cdot$ BLUTIGE-ERNST \\ 1. JAHR SATIRISCHE WOCHENSCHRIFT. HERAUSGEBER: CARL EINSTEIN, GEORGE GROSZ. \\ PFENNIG}

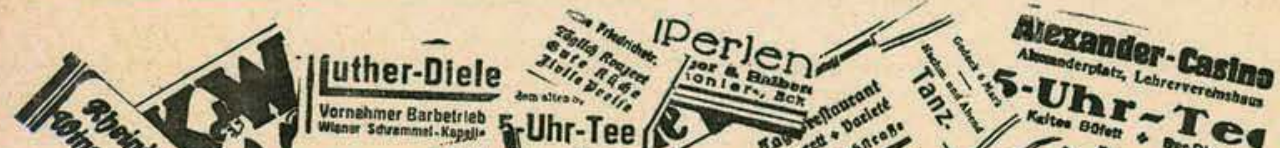

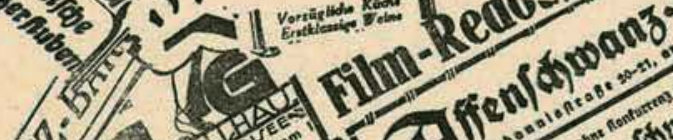

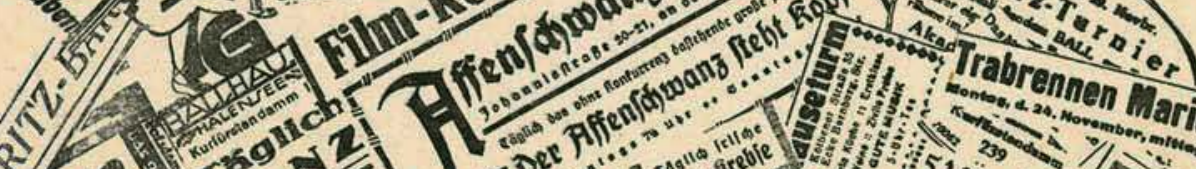

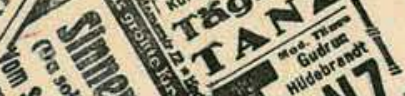

20.

Det

OP 120

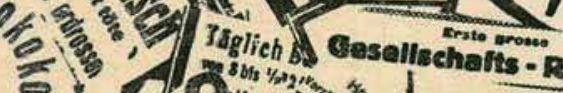

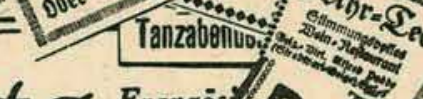

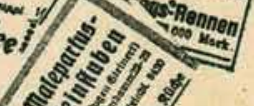

172 Franzos 0 Stras 0
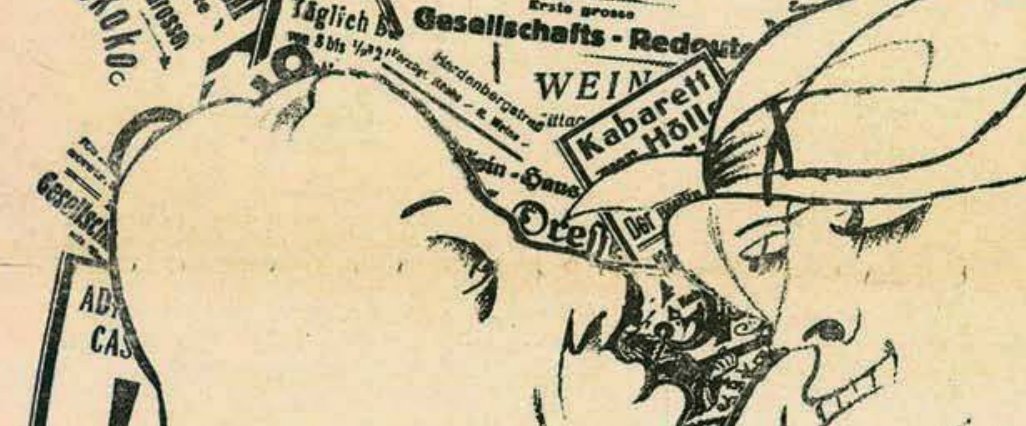
Le texte "Anmerkungen " est une critique à peine masquée de cet idéalisme immaculé que fut l'expressionnisme activiste, tel qu'il se constituait et se propageait à travers les pages de Die Aktion même, revue ouverte à différents courants anarchistes et antimatérialistes de gauche7. Mieux, la cible précise d'Einstein était sans aucun doute Ludwig Rubiner, essayiste influent au sein de l'expressionnisme, avec lequel il allait engager plus tard une polémique ouverte ${ }^{8}$. Quelques numéros avant celui où paraissait "Anmerkungen", Rubiner avait écrit le texte emphatique "Le poète se saisit de la politique» ("Der Dichter greift in die Politik»), menant un paragone assez âpre entre le poète et le politique. Il exaltait l'efficacité de l'action du poète et du peintre, dont les œuvres prouvaient, selon lui, qu'une subjectivité forte était capable de détruire les formes sclérosées du réel par la simple extériorisation de son "intensité». Ne faisant donc aucune différence entre la représentation et la réalité, qu'il considérait de toute évidence comme également malléables, Rubiner écrivait : «La révolution est là. L'humanité, le formateur vont contre la dure habitude, contre le ventre du monde. Il n'y a plus de matière9. " Le matérialisme visé ici était autant celui du conformisme capitaliste que celui de la révolution marxiste. «Les Idées sont toujours là, et toujours nouvelles. Et chaque Idée est une catastrophe », poursuivait Rubiner ${ }^{10}$. Mais c'était sans doute parce que la « catastrophe " ne pouvait pas être suscitée par l'Idée, "toujours là, toujours nouvelle", qu'Einstein poursuivit sa riposte contre Rubiner. Voulant briser le solipsisme expressionniste, il écrivit un deuxième texte au titre complété : «Politische Anmerkungen», c'est-à-dire «Notes politiques ».

L'intention de ce texte était donc positive, car il devait expliquer la révolte subjective à vocation politique telle qu'Einstein la concevait lui-même. À l'instar de Walter Benjamin, qui écrivit à la veille d'une autre guerre : «Que les choses continuent comment avant : voilà la catastrophe ${ }^{11}$ ", Einstein estimait que si dans le cadre démocratique libéral les choses avaient l'air « lisses et polies, avec des belles graduations", "vu de près, tout se déroule de manière catastrophique, [...]. Les catastrophes - qu'est-ce qu'elles sont rationnelles ${ }^{12}$ ». Nous l'avons dit : l'illusionnisme esthétique de la démocratie consistait dans l'occultation de sa catastrophe permanente sous les belles proportions du consensus. Mais, selon Einstein, cette catastrophe, sourde et invisible, devait être exposée au grand jour et arrêtée par une catastrophe volontaire et dotée d'une évidence écrasante. Cette catastrophe sensible, censée constituer l'objet de l'expérience humaine et ayant donc lieu dans l'histoire, serait complètement différente bien sûr de celle invoquée par l'expressionnisme activiste. Car si pour le «révolteur» l'histoire était «insignifiante, et même fausse en soi ", pour Einstein pouvait « donner forme à l'histoire [Geschichtebildend] l'homme qui donne plus que l'équilibre des antithèses [...]. Je ne veux pas dire l'original, ce colporteur ennuyeux, cet irréligieux, qui n'ignore même pas sans peur que nous restons à l'intérieur des limites données par Dieu, mais le vigoureux, celui dont la bouche s'ouvre comme la terre fendue, le révolutionnaire en tant que tel ${ }^{13}$."

Le nietzschéisme d'Einstein a été une composante déterminante de sa pensée ${ }^{14}$. Ses cibles, en art comme en politique, ont toujours été en fin de compte des types «ré-actifs», agis plutôt qu'agissants, nihilistes inavoués plutôt que créateurs, rancuniers plutôt que généreux : Rubiner en faisait évidemment partie. Comme pour le Nietzsche de la Deuxième Considération intempestive, l'homme qui peut «donner forme à l'histoire » était pour Einstein le contre-modèle de l'historiciste - du social-démocrate, qui croyait ferme en la continuité de l'histoire, ou du bour-
-

7. La lecture très critique de l'expressionnisme par Einstein devait constituer une constante dans sa pensée. II expliquait l'abstraction expressionniste, en particulier, d'une manière assez proche de celle qui s'appliquait à Rubiner : comme une «théologie négative ». Sur ce point, $c f$. la traduction dans ce numéro du chapitre consacré au Cavalier bleu dans Die Kunst des 20. Jahrhunderts. Cf. p. 198-229.

8. Dans les textes «Brief an Ludwig Rubiner » [《Lettre à Ludwig Rubiner ) et « Journalismus am falschem Ort » (《 Journalisme mal placé»; 1914), publiés également dans Die Aktion, Einstein accusait Rubiner d'attribuer une dimension «polémique » à la peinture. Ce dernier estimait en effet que Robert Delaunay était un peintre «polémique » dans la mesure où il n'était pas un peintre «créatif». La logique nietzschéenne d'Einstein rejetait toute conception ré-active de l'art au profit d'une conception altière, parce qu'intrinsèquement positive.

9. Ludwig Rubiner, « Der Dichter greift in die Politik, \| », Die Aktion II, 23, 5 juin 1912, p. 709-712 ; ici p. 709.

10. Ibid.

11. Voici la thèse de Benjamin dans son intégralité : « II faut fonder le concept de progrès sur l'idée de la catastrophe. Que les choses continuent comme avant: voilà la catastrophe. Elle ne réside pas dans ce qui va arriver, mais dans ce qui, dans chaque situation, est donné. Ainsi Strinberg écrit-il - dans Le Chemin de Damas : l'enfer n'est pas quelque chose qui nous attend, mais la vie que nous menons ici. » Walter Benjamin, Paris. Capitale du XIx siècle. Le livre des passages, trad. Jean Lacoste, Paris, Éditions du Cerf, 1989, p. 491.

12. Carl Einstein, «Politische Anmerkungen », art. cit., p. 143.

13. Ibid., p. 144.

14. Sebastian Zeidler insiste beaucoup sur le nietzschéisme d'Einstein dans son écrit « Form as Revolt. Carl Einstein's Philosophy of the Real and the Work of Paul Klee », RES. Anthropology and Aesthetics 57/58, 2010, p. 229-263. 


\section{CARL EINSTEIN ET LES PRIMITIVISMES}

-

15. Carl Einstein, « Politische Anmerkungen », art. cit., p. 144.

16. Ibid., p. 145.

17. Ibid., p. 144.

18. Georges Didi-Huberman, Devant le temps : histoire de l'art et anachronisme des images, Paris, Éditions de Minuit, 2000, p. 176.

19. Sebastian Zeidler, « Life and Death from Babylon to Picasso: Carl Einstein's Ontology of Art at the Time of Documents », Papers of Surrealism?, http://www.surrealismcentre. ac.uk/papersofsurrealism/ journal?/acrobat\%20files/articles/ Zeidlerpdf.pd.

20. Tenter de comprendre l'antihumanisme einsteinien impliquerait par exemple de ne pas redouter sa comparaison à l'antihumanisme d'un T.E. Hulme, penseur réactionnaire s'il en est, mais qui a défendu des thèses assez similaires à celles d'Einstein concernant l'optimisme humaniste et la démocratie. Comme Einstein, il a associé le primitif au renversement de cette dernière (ce philosophe était proche du milieu vorticiste anglais]. Georges Sorel a été une référence commune aux deux penseurs. Cf. T.E. Hulme, Speculations. Essays on Humanism and the Philosophy of Art, Londres-New York, Routledge and Kegan Paul, 1987. Une telle comparaison ne viserait pas à démontrer qu'Einstein a été un réactionnaire, mais à historiciser son besoin d'une rupture du temps en en restituant les ingrédients nostalgiques. Faute d'espace, nous nous limiterons pour le moment à cette remarque. geois cultivé (Bildungsbürger), sujet artificiellement composé des "meilleurs» moments du passé. Et, comme pour le Nietzsche de la Volonté de puissance, l'art devait, selon Einstein, accroître la force d'action du sujet au lieu de l'apaiser ou de l'inhiber.

Du côté de la négation, il y avait donc l'art imitatif, l'historicisme, le libéralisme démocratique. L'art reproduisait les objets, l'histoire les faits, la démocratie libérale les rapports du pouvoir. Et cette répétition du même se faisait, chaque fois, grâce au dispositif d'illusion qui, en l'enveloppant, la soustrayait aux regards : le beau, le classique, le progrès, la démocratie et tout cela à la fois. Mais, du côté de l'affirmation, il y avait le "primitif». Allant bien au-delà de ses manifestations habituelles du populaire, de l'archaïque et du tribal, le «primitif» désignait chez Einstein "l'homme univoque, qui doit agir, que ce soit dans les livres, dans les tableaux, n'importe où. Nous en avons assez des dialecticiens, des comédiens et des artistes ascétiques (ces agneaux blancs) - nous exigeons des livres, qui renforcent et organisent les actions; des tableaux, sans les inhibitions des costumes corrupteurs, qui renforcent l'histoire ${ }^{15}$." Renoncer aux costumes de l'histoire au profit d'une mise à nu primitive, cela exigeait un certain héroïsme, non pas pathétique, mais sec et impassible. Ainsi, Einstein décrivait-il plus loin le primitif comme "un appauvrissement, mais aussi une critique active de tout immondice, qu'on ne refuse pas, mais qu'on écarte sans émotion. Il vaux mieux rester sans rien et s'en tenir à la pauvreté convenable que de continuer dans le superflu ${ }^{16}$. Mais cette pauvreté volontaire du type primitif n'était que la base sur laquelle il devait construire sa véritable identité : l'unité nietzschéenne de la "forme » et de la «force " (Form und Gewalt), le domptage, par la rigueur d'une raison impeccable dans son fonctionnement, de son désir de liberté. Maîtriser l'intensité de la passion, sans la neutraliser, canaliser "l'hystérie féminine» diffuse en la transformant en force "virile", c'était là aussi la vision très «mâle » du primitif par Einstein. Dans tous les cas, univoque et intransigeant, le primitif était pour lui une catégorie ontologique universelle, qui trouvait ses actualisations dans tous les champs de la vie : il se trouvait aux antipodes de l'équivocité perceptive de l'impressionnisme et cognitive du symbolisme mystique pour les mêmes raisons qu'il se trouvait aux antipodes de l'équivocité intellectuelle et morale de la législation démocratique, dépourvue de tout fondement métaphysique : "Ce qui nous manque : des forces univoques, intransigeantes. Mais celles-ci dérivent toujours du religieux, bien plus, ce dernier a été jusqu'à présent le seul refuge des idées ${ }^{17}$. » La lutte d'Einstein contre la mimèsis esthétique et politique était une expression de son antihumanisme et de son antirationalisme, elle était l'expression de sa volonté de voir la vie humaine instaurée à nouveau sur le respect des valeurs absolues, capables de prendre la relève de la religion. Cette nostalgie d'Einstein à l'égard de la religion, la même qui l'avait fait condamner l'irréligiosité de Rubiner en lui rappelant les limites de l'homme fixées par Dieu, a été évacuée des études qui lui ont été consacrées. Georges Didi-Huberman a souligné son antihumanisme, mais pour le dissocier de toute pensée désenchantée ${ }^{18}$. Et Sebastian Zeidler a attaqué cette interprétation, considérant que ce nietzschéen qu'était Einstein ne pouvait pas être réduit à l'anti-, parce qu'il était avant tout un penseur de l'invention et de la création $^{19}$. Mais ces deux lectures se sentent tenues d'émettre des jugements de valeur sur leur objet. Une lecture "par-delà bien et mal " (le nietzschéisme est décidément multiple!) peut rester ouverte à la complexité de son objet dans ce qu'elle détient de plus admirable mais aussi de plus ambigu ${ }^{20}$. 
Le mythe social de Georges Sorel

L'une des actualisations les plus puissantes du religieux dans le monde moderne, auquel appartenait Einstein, était la révolution dans sa capacité à signifier la rupture du temps et à véhiculer la dialectique apocalyptique de la destruction et de la construction. Dans son étude magistrale de la conception de l'histoire moderne comme une histoire du salut, Karl Löwith a analysé la révolution marxiste comme une sécularisation de l'Apocalypse $\mathrm{e}^{21}$. Mais si le rationalisme marxiste présupposait l'occultation des sources de son eschatologie politique, sa révision impliquait, au contraire, leur puissante actualisation. C'est exactement ce que faisait Einstein, en poursuivant sur ce point l'entreprise intellectuelle de Sorel, qui pensait, comme lui, qu'on "peut indéfiniment parler de révoltes sans provoquer jamais aucun mouvement révolutionnaire, tant qu'il n'y a pas de mythes acceptés par les masses ${ }^{22}$ ». La nécessité d'inventer un "mythe social» collectif, mettant un terme à l'écoulement tranquille de l'histoire grâce à sa nature imageante, motrice, irréfutable et indécomposable; la réalité comme un enchaînement pessimiste d'obstacles, que seule la catastrophe pouvait briser au grand dam de tout optimisme béat : ce furent là deux thèses de Sorel, développées essentiellement dans ses Réflexions sur la violence, dont les pages de la revue Die Aktion se firent régulièrement l'écho et que l'on retrouve chez Einstein ${ }^{23}$.

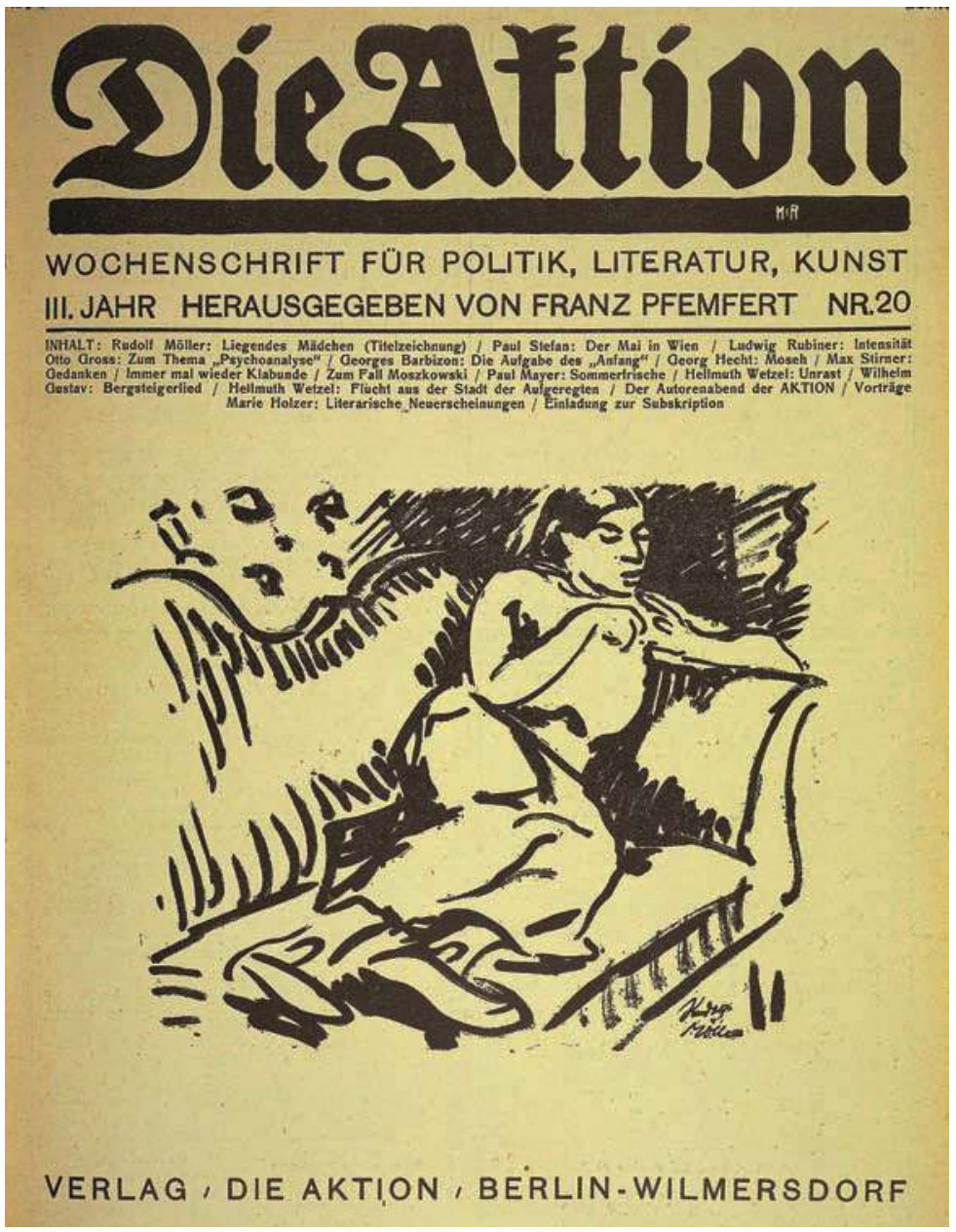

- $\bullet$

21. Cf. Karl Löwith, Histoire et Salut. Les présupposés théologiques de la philosophie de l'histoire, Paris, Gallimard, 2002.

22. Georges Sorel, Réflexions sur la violence, Paris-Genève, Ressources, 1981, p. 37.

23. Je remercie Sebastian Zeidler, qui a été un interlocuteur de choix sur la référence sorélienne de la pensée de Carl Einstein.
Fig. 4 Die Aktion. Wochenschrift für Politik, Literatur, Kunst , édition de Franz Pfemfert, 1913 / cahier $n^{\circ}$ 2O, Berlin @ BPK, Berlin, dist. RMN / Dietmar Katz. 
- $\bullet$

24. Georges Sorel, op. cit., p. 26.

25. Ibid., p. 15.

26. Ibid., p. 146

27. «Le plus ancien programme systématique de l'idéalisme allemand », dans Philippe LacoueLabarthe et Jean-Luc Nancy, L'Absolu littéraire, Paris, Seuil, 1978, p. 54.
Sorel était convaincu de la puissance d'entraînement inhérente à son « mythe social " pour mobiliser les prolétaires dans la lutte des classes que l'eudémonisme et le pacifisme sociaux-démocrates avaient considérablement ramollie. Pour Sorel, l'action sur le présent nécessitait sa projection identificatoire dans l'avenir. Le mythe, conçu comme une image mentale, devait donc présenter comme déjà achevé ce qui ne l'était pas encore : en l'occurrence, la grève générale en tant que "répétition " préparant la révolution à venir. Il écrivait : "Les hommes qui participent aux grands mouvements sociaux se représentent leur action prochaine sous forme d'images de batailles assurant le triomphe de leur cause. Je proposais de nommer mythes ces constructions dont la connaissance offre tant d'importance pour l'historien. La grève générale des syndicalistes et la révolution catastrophique de Marx sont des mythes ${ }^{24}$. " La force motrice du mythe sorélien se mesurait à l'aune de l'adhésion qu'il pourrait susciter. Une chose était sûre : la guerre à laquelle appelait ce mythe contre la social-démocratie et la bourgeoisie n'était pas matérialiste, car ce qui l'emportait, chez Sorel, c'était l'effet de transfiguration sublime que le mythe saurait exercer sur les masses plutôt que sa réalisation concrète. Comme chaque fois que l'homme était appelé à devenir plus grand que lui-même, il s'agissait là encore d'une " marche vers la délivrance ${ }^{25}$ ". Pour Sorel, les premiers chrétiens correspondaient à l'archétype de cette marche, eux qui, malgré les persécutions, ont durablement enraciné le christianisme grâce à l'espoir fondé dans l'attente de l'Apocalypse, qu'ils croyaient toute proche. Rien ne leur prouvait, il est vrai, son irruption imminente, mais la seule chose qui comptait était qu'ils y croyaient. Car ce que Sorel appréciait dans le mythe, cétait avant tout cette efficacité que lui donnaient son caractère d'image, sa forme synthétique plutôt qu'analytique, son adresse à l'affect plutôt qu'à la raison. Dans la lutte du syndicalisme révolutionnaire contre la social-démocratie et la bourgeoisie aux instincts émoussés, il fallait non pas «atténuer les oppositions, mais [...] les mettre en relief ", il fallait « donner un aspect aussi solide que possible aux groupements qui luttent entre eux ». Et Sorel d'ajouter : "On représentera les mouvements des masses révoltées de telle manière que lâme des révoltés en reçoive une impression pleinement maîtrisante. Le langage ne saurait suffire pour produire de tels résultats d'une manière assurée; il faut faire appel à des ensembles d'images capables d'évoquer en bloc et par la seule intuition, avant toute analyse réfléchie, la masse des sentiments qui correspondent aux diverses manifestations de la guerre engagée par le socialisme contre la société moderne ${ }^{26}$."

En vérité, le bergsonisme de Sorel lui permettait de revisiter la thèse principale de l'éloge du mythe dans la métaphysique moderne, des romantiques à Friedrich Nietzsche en passant par Richard Wagner, à savoir sa supériorité supposée face à la science et à la « raison abstraite ». Là où les romantiques avaient parlé de "poésie ", Sorel parlait d'«image ». Pour le romantisme et l'idéalisme allemands, c'était en tant que poésie en effet que le mythe pouvait suppléer à l'entendement pour réussir la formation de la communauté nouvelle. Les trois auteurs probables du Plus ancien programme systématique de l'idéalisme allemand - Hegel, Schelling et Hölderlin - interprétaient à leur façon la leçon schillérienne d'une "éducation esthétique de l'homme » en préconisant une "nouvelle mythologie " qui, contrairement à la mythologie indistincte des commencements, serait au service des Idées, de la Raison ${ }^{27}$. Cette mythologie aurait permis de donner un caractère "sensible", "esthétique " aux Idées, afin que le peuple, peu enclin aux subtilités de l'esprit, puisse s'y intéresser. Car, comme Schelling devait l'expliquer quelques décennies plus tard dans sa Philosophie de la mythologie, l'adéquation 


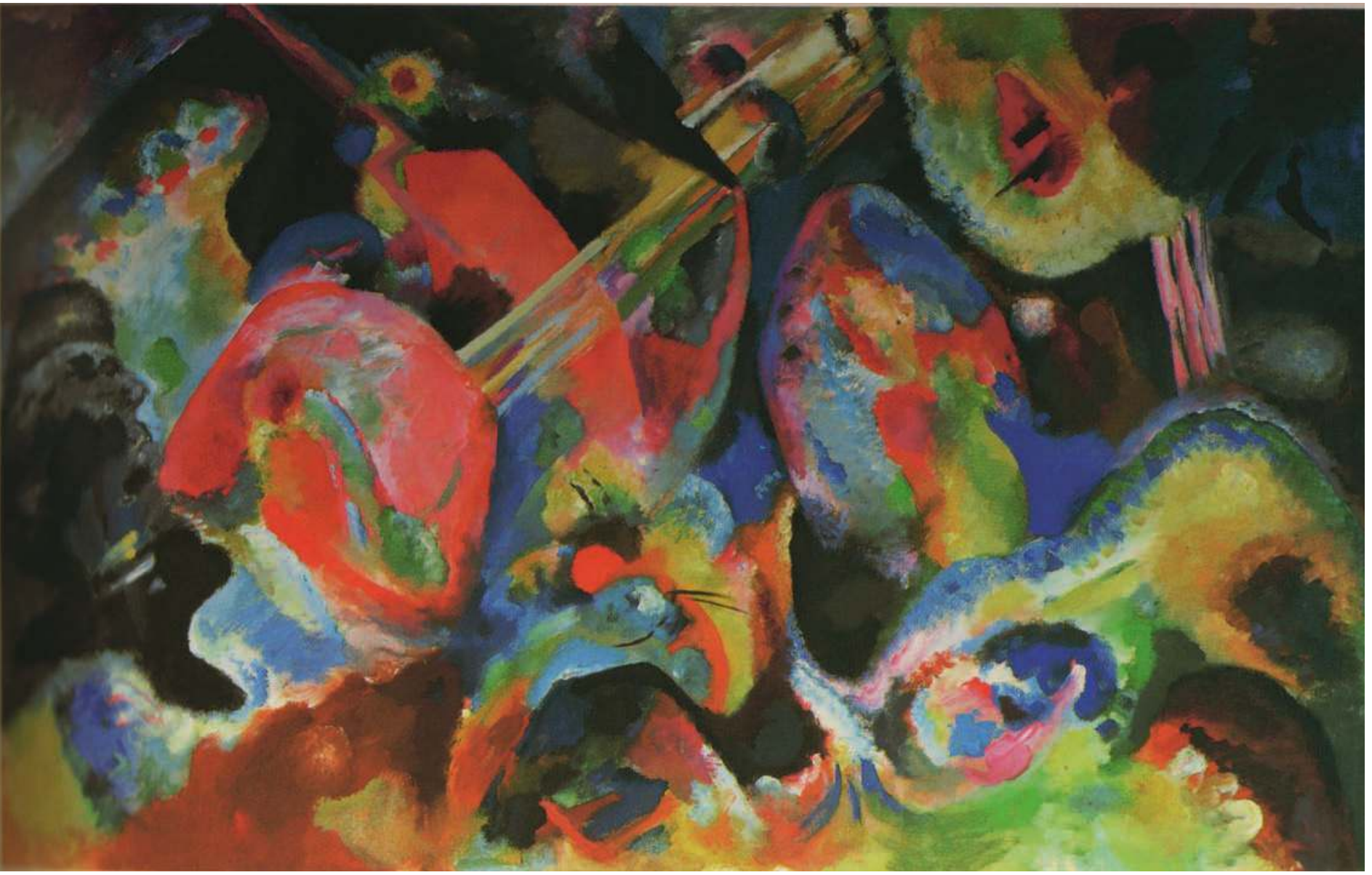

Fig. 5 Vassily Kandinsky, Improvisation. Déluge, 1913, Lenbachhaus, Munich ๔ ADAGP, Paris 2011. 


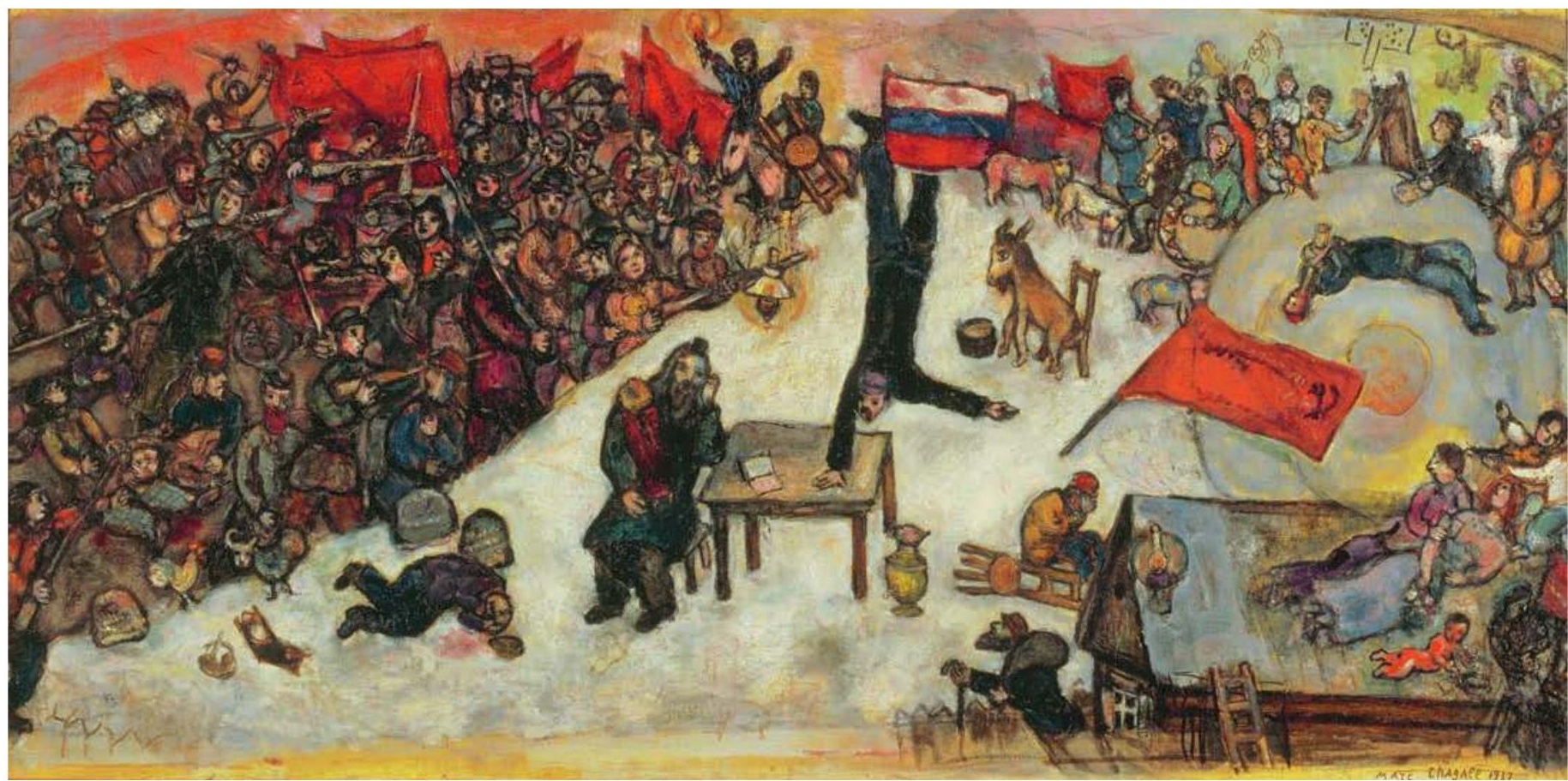

Fig. 6 Marc Chagall, Esquisse pour La Révolution, 1937. Collection Centre Pompidou, dist. RMN / Philippe Migeat $\odot$ ADAGP, Paris 2011, Marc Chagall ${ }^{\text {. }}$.

-

28. F.W.J. Schelling, Introduction à la philosophie de la mythologie, t. I, Paris, Aubier, 1946, p. 237.

29. Ibid.

30. Cf. Georges Sorel, Réflexions sur la violence (Introduction/Lettre à Daniel Halévy), op. cit., p. 37-39. Sur la distinction entre mythe et utopie chez Sorel, cf. Éric Michaud, «Le mythe social ou l'efficacité de l'image sans images », Mil neuf cent. Revue d'histoire intellectuelle 28 (numéro spécial « Les foules et la démocratie »), 2010, p. 173-183. parfaite entre la forme et le contenu, entre le signifiant et le signifié du mythe, pouvait transmettre mimétiquement les idées les plus élaborées ${ }^{28}$. Rejetant donc toute interprétation allégorique, qui faisait en fin de compte du mythe une conséquence de l'intentionnalité rationaliste, Schelling forgeait le néologisme "tautégorie" pour définir son incomparable force mimétique : "La mythologie n'est pas allégorique; elle est tautégorique. Pour elle les dieux sont des êtres qui existent réellement, qui ne sont rien d'autre, ne signifient rien d'autre, mais signifient seulement ce qu'ils sont. Autrefois on opposait sens propre et sens doctrinal. Mais, d'après notre explication, l'un est inséparable de l'autre, et au lieu de sacrifier le sens propre à une signification doctrinale ou de vouloir sauver [...] le sens propre aux dépens de la signification doctrinale, nous croyons [...] devoir affirmer l'unité, l'inséparabilité de l'un et de l'autre ${ }^{29}$. " Sens propre et sens figuré, particulier et universel, esthétique et raison coïncidaient dans la tautégorie mythique, qui s'avérait être ainsi une sorte d'archi-mimèsis se célébrant au sein de la conscience subjective.

C'était cette même efficacité qu'évoquait Sorel quand il écrivait dans sa lettre à Daniel Halévy qu'«en employant le terme du mythe, je croyais avoir fait une heureuse trouvaille, parce que je refusais ainsi toute discussion avec les gens qui veulent soumettre la grève générale à une critique de détail ${ }^{30}$ ». Les armes du mythe social contre l'utopie, dans son versant marxiste autant que réformiste, étaient bien affûtées : si l'utopie discutait, le mythe social voulait; si l'utopie décrivait, le mythe affirmait; si l'utopie devait argumenter pour convaincre, toute énonciation mythique était la preuve même de sa vérité. La conception qu'Einstein se faisait du "primitif ", dans son opposition à la démocratie parlementaire et à l'utopie socialiste, expressions de l'optimisme humaniste qu'il honnissait, était imprégnée par le mythe sorélien. Son « univocité » et son «intransigeance », 
l'accusation de ses traits et son intensité formelle correspondaient aussi de manière exacte à l'image motrice du mythe, devant suggérer l'urgence du conflit et faire oublier, par-là même, les discussions lénifiantes des assemblées démocratiques et des utopies réformistes. Mais surtout, le principe "primitif» d'Einstein était lié à la même action temporelle que Sorel avait préconisée pour son mythe social : en rappelant la révolution à ses origines religieuses, le primitif devait accélérer l'éclatement du trop-plein de l'histoire par une catastrophe. "Le primitif, écrivait Einstein, est capable de sauter par-dessus toutes nos têtes, [en ne se dirigeant pas] vers une utopie, mais vers une force violente; non pas vers un ordre accommodant, mais vers un Jugement ${ }^{31}$. Ce Jugement, rupture de la causalité accommodante au profit de l'instant, actualisation de ce que fut autrefois la transcendance divine sous la forme de la révolution éclatant au sein du sujet, Einstein a pu en avoir l'avant-goût en tant que spectateur de la sculpture africaine.

\section{Indifférence souveraine : fin de la démocratie et révolution à rebours}

Einstein a écrit son traité Negerplastik en 1914, mais il ne l'a publié qu'en pleine guerre et alors qu'il était lui-même au front, en 1915. Ce traité était composé d'un texte de quelques pages et d'un ensemble d'images de sculptures africaines, qu'Einstein n'a pas commentées nommément et de manière spécifique. Il y a une contradiction logique dans le postulat méthodologique de ce texte, à laquelle il nous faudra revenir plus tard. Disons à titre provisoire qu'Einstein critiquait le perspectivisme des lectures antérieures - passablement dépréciatives - de l'art primitif, mais pour revendiquer quelques lignes plus loin le "présentisme " de sa propre approche : "Cette brève description de l'art africain, écrivait-il, ne pourra se soustraire aux expériences faites par l'art contemporain, d'autant plus que ce qui prend de l'importance historique est toujours fonction du présent immédiat ${ }^{32}$. " De fait, cette urgence du présent, pas seulement celui des artistes modernes, mais aussi celui d'Einstein lui-même en tant que médiateur entre l'art et la politique de son temps, déterminait profondément son explication de la sculpture africaine.

Comme tous les auteurs qui, à partir de la fin du xviII siècle, ont adopté des thèses primitivistes, Einstein considérait la sculpture africaine comme un contre-modèle du classique tel qu'il a prévalu dans l'art occidental, à quelques exceptions près, à partir de la Renaissance ${ }^{33}$. Façonnées dans les conditions objectives procurées par la religion, les lois visuelles qui opéraient dans la sculpture nègre ne pouvaient que différer considérablement de celles qui avaient dominé l'art de l'Occident rationaliste. Mais là où la problématique d'Einstein devenait plus singulière, sans pour autant se démarquer des fondamentaux du discours primitiviste classique, c'était lorsqu'il commençait à expliquer les lois de vision propres à la sculpture nègre.

Ces lois se fédéraient autour d'un principe central, celui de l'« autonomie » de sa forme. Autonomie et indifférence souveraine envers son adorateur trouvaient, pour cet objet cultuel qu'était la sculpture africaine, une même explication : " $\mathrm{Ce}$ qui caractérise les sculptures nègres, c'est une forte autonomie des parties; ceci aussi est fixé par une règle religieuse. L'orientation de ces parties est fixée non en fonction du spectateur mais en fonction d'elles-mêmes; elles sont ressenties à partir de la masse compacte, et non avec un recul qui les affaiblirait ${ }^{34}$. " La sculpture occidentale se trouvait aux antipodes de cette «compacité» impassible.

\section{- $\bullet$}

31. Carl Einstein, «Die

Sozialdemokratie » (publié en 1914 dans Die Aktion ], Werke, t. I, op. cit., p. 213.

32. Id., La Sculpture nègre, trad. Liliane Meffre, Paris, L'Harmattan, 1998, p. 18-19.

33. Contrairement à la lecture de Georges Didi-Huberman, il ne nous semble pas qu'il faille dissocier Einstein de tout primitivisme. Le primitivisme ne se réduit pas à l'exotisme, ni à des démarches purement nostalgiques. II n'est pas un phénomène purement et simplement négatif. Cf. Christian Marouby, Utopie et primitivisme. Essai sur l'imaginaire anthropologique à l'âge classique, Paris, Seuil, 1990. Par ailleurs, malgré la singularité de son approche, Einstein conserve plusieurs thèmes primitivistes dans son texte. Nous essayons ici de montrer que l'interprétation de la sculpture africaine était une manière de commenter la platitude de la démocratie moderne, mais aussi le régime de servitude qu'elle imposait. Zoë Strother donne également dans ce même numéro une autre interprétation du primitivisme de Negerplastik, p. 30-55.

34. Carl Einstein, La Sculpture nègre, op. cit., p. 30. 


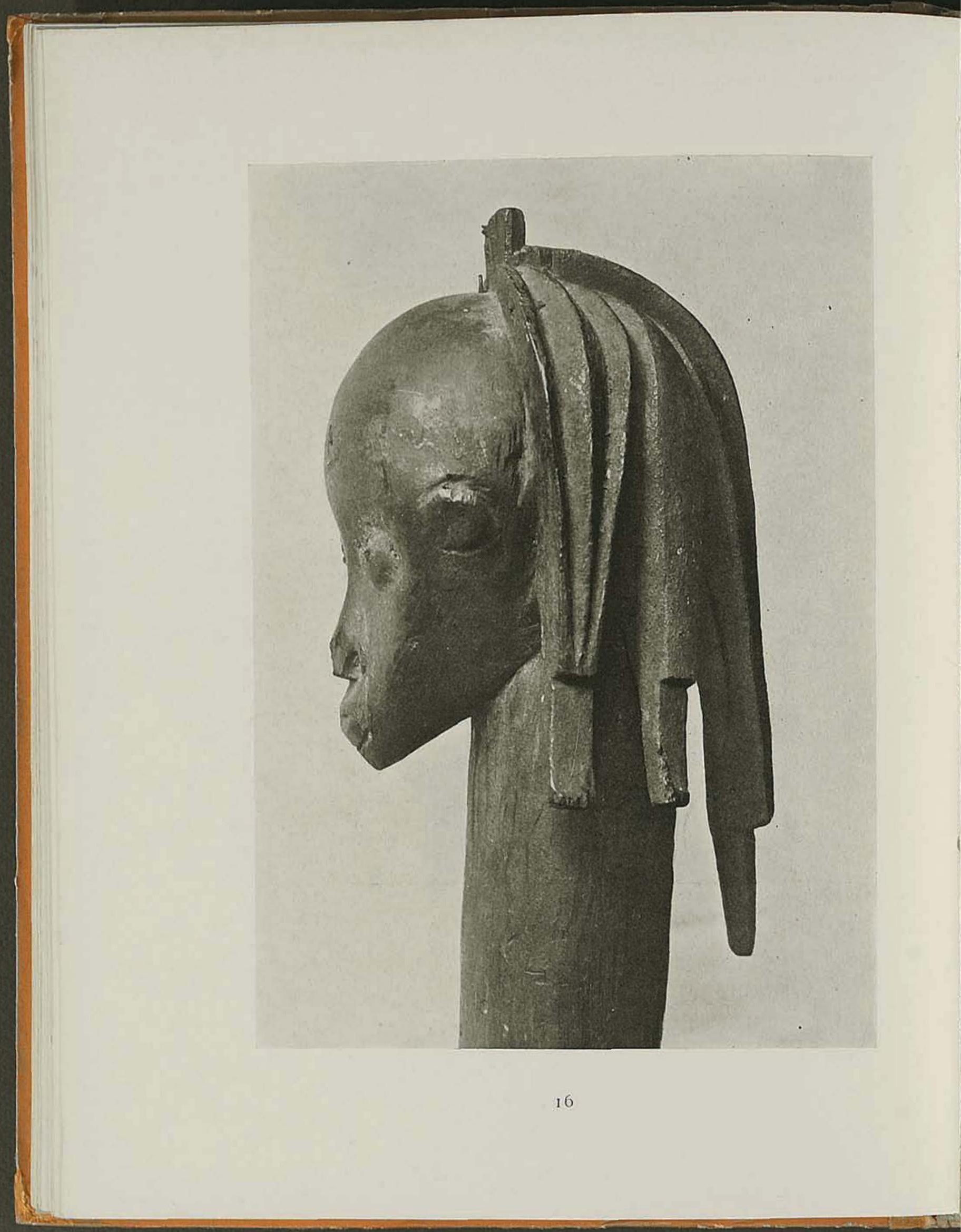




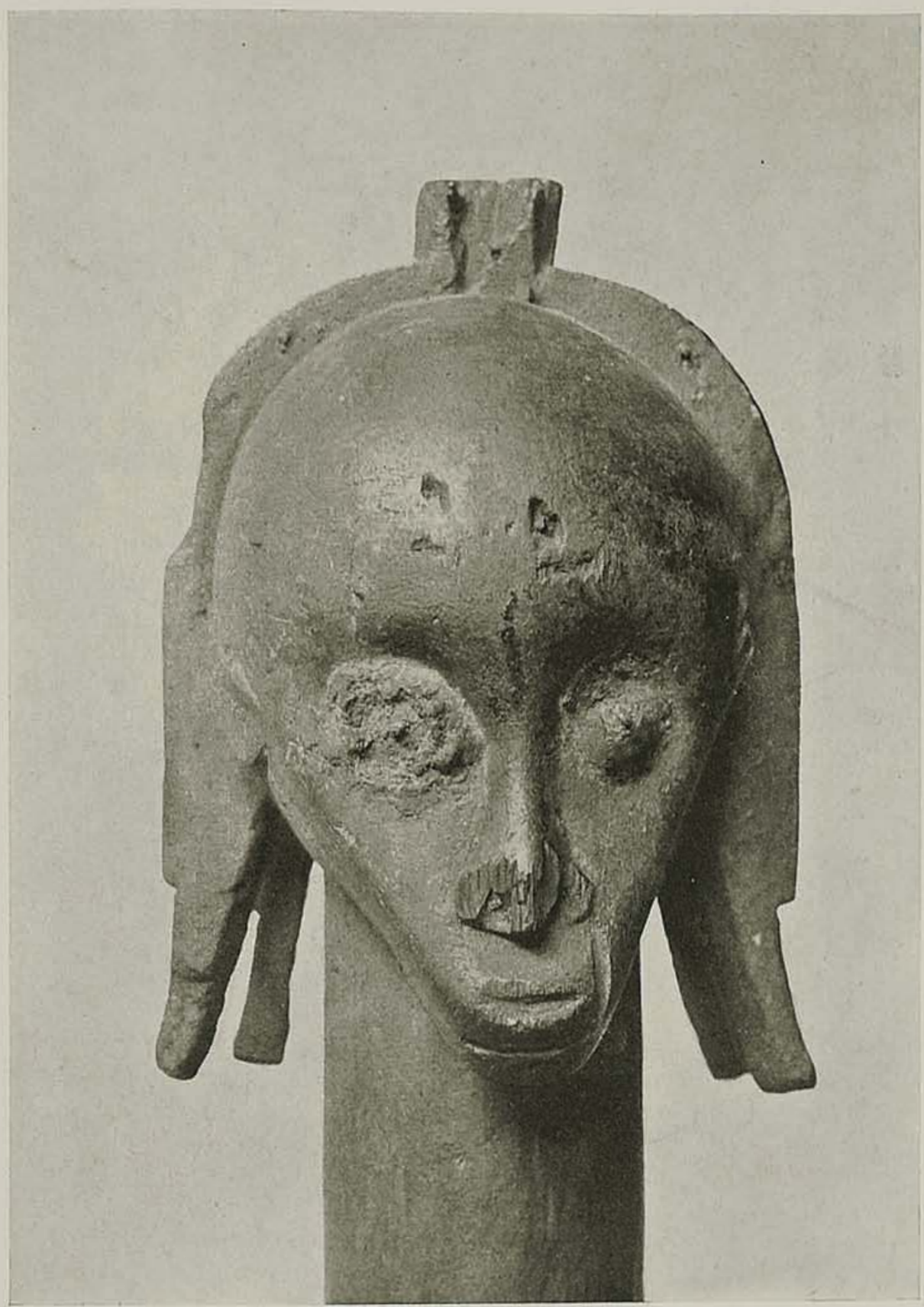




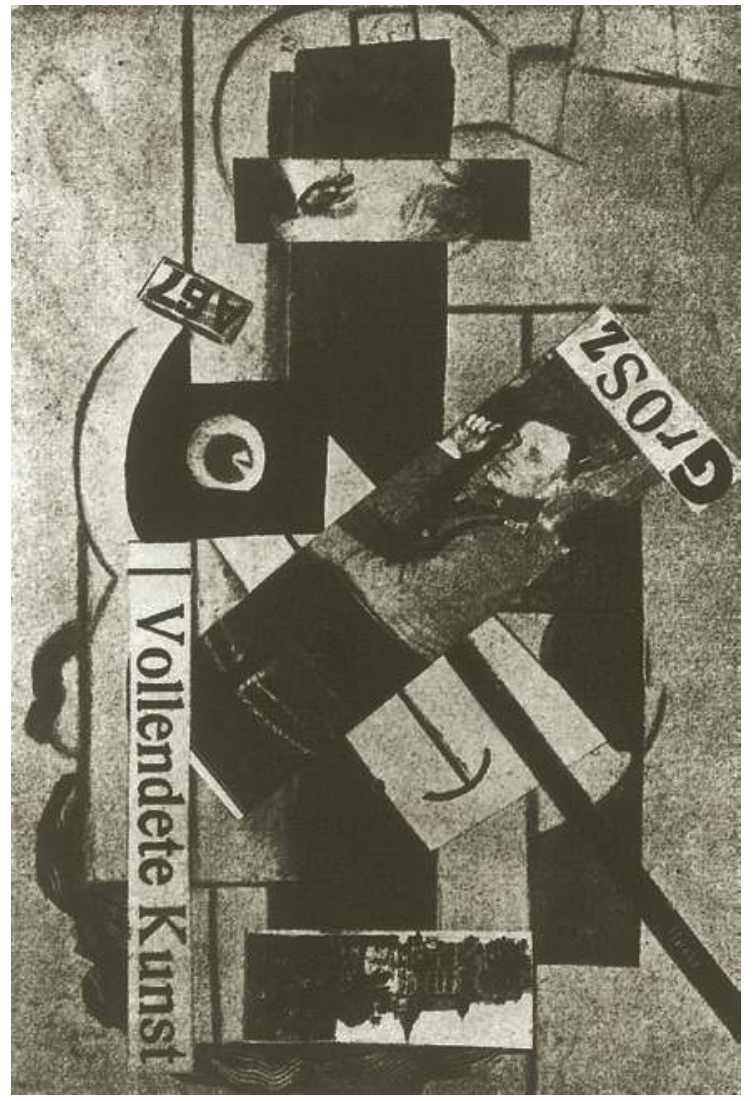

Fig. 8 George Grosz et John Heartfield, Corrected Picasso. La Vie heureuse (dedicated to Dr. Karl Einstein), collage perdu, reproduit dans le catalogue de l'exposition Dada Fair, New York, 1920 (c) The Heartfield Community of Heirs / ADAGP, Paris 2011

PAGE 68

Fig. 7 Carl Einstein, Negerplastik, planches 16-17: Fang, Gabon, tête de reliquaire nlo byeri. Photo Alberto Ricci.

- -

35. Ibid.

36. Ibid., p. 26.

37. Cf. Martin Jay, « Modernism and the Specter of Psychologism », Modernism/modernity, III, 2, 1996, p. 93-111.

38. Carl Einstein, La Sculpture nègre, op. cit., p. 28.

39. Ibid.

40. Ibid., p. 31
Sa nature "plastique" avait même souffert d'une contamination par le "pictural». La perspective, le mouvement de la forme, le modelé, la "main de l'artiste " étaient autant de caractéristiques éminemment picturales qu'Einstein relevait dans la sculpture moderne. Un paragone se profile ici : la peinture, art de l'intériorité, de la mobilité et produit de cette autonomie négative que la sécularisation avait léguée à l'individu et à son art, contaminait la sculpture, art dont la compacité et le caractère public ont nourri systématiquement l'imaginaire cultuel nostalgique des temps modernes. La peinture faisait œuvre de dissolution - de la forme, de la communauté, du temps. La sculpture africaine venait arrêter cette dynamique centrifuge, mais sans céder pour autant à une rigidité sclérosée. Ordonnée autour de quelques points centraux du volume en accentuant la frontalité de la figure sans renoncer à sa tridimensionnalité spatiale mais faisant d'elle sa composante à part entière, la sculpture nègre savait saisir le spectateur par son «intensité architecturée ", qui était sur le plan de la qualité ce que la monumentalité moderne aspirait à être par la quantité35.

Mais surtout, la sculpture nègre ne cherchait guère à nouer un dialogue quelconque avec son adorateur de jadis et son spectateur d'aujourd'hui. Car, selon Einstein, le maitre de la sculpture occidentale était le spectateur plutôt que l'artiste, qui ne cherchait après tout qu'à satisfaire les attentes du premier. Perfidement introduit dans la forme, jusqu'à en devenir un élément génétique, le spectateur a entraîné la temporalisation sculpturale : «La sculpture était objet de dialogue entre deux personnes. Ce qui devait avant tout intéresser un sculpteur avec une pareille orientation, c'était de déterminer à l'avance l'effet et le spectateur; pour anticiper l'effet et le tester, il fut amené à s'identifier au spectateur [...] et les sculptures doivent alors être considérées comme des périphrases de l'effet produit. Le facteur psychotemporel l'emporta complètement sur la détermination de l'espace ${ }^{36}$.» La tyrannie du spectateur était, pour Einstein, une expression de la tyrannie, plus vaste, de l'humanisme dans ce qu'il a pu engendrer de pire : le psychologisme ${ }^{37}$. La sculpture nègre, cependant, "ne se mêlera jamais au devenir humain et, si elle le faisait, ce serait en tant que souveraine et, une fois de plus, en gardant ses distances ${ }^{38}$ ". Si la sculpture africaine affirmait sa spatialité propre, fermée sur elle-même et dégagée de tout lien, cela était dû au fait que sa création et sa réception étaient des moments du processus unique de la vénération de dieu : «L'œuvre est créée dans l'adoration, dans la crainte de dieu et ce sont les mêmes effets qu'elle provoque ${ }^{39}$. " En somme, la sculpture nègre opposait l'unité à la séparation, l'espace au temps, l'être au devenir et, bien sûr, l'efficacité mythique à l'effet psychologique : «Elle ne signifie rien, elle n'est pas symbole; elle est le dieu qui conserve sa réalité mythique close, dans laquelle il inclut l'adorateur, le transforme lui aussi en être mythique et abolit son existence humaine ${ }^{40}$. " Loin de se laisser assimiler aux humeurs changeantes de l'homme, la sculpture africaine absorbait ce dernier dans sa propre absence de temps : dans l'atemporalité mythique.

Cela est frappant : tous les prédicats de la sculpture africaine dans ce texte renvoient exactement et infailliblement à ce qu'Einstein appelait dans ses écrits 
politiques le "primitif", et dans d'autres écrits de la même période, plus purement spéculatifs, la « totalité » ou la « loi ». La « loi [Das Gesetz], avait-il écrit dans un texte homonyme, est arbitraire. Elle est un moment productif qui se dérobe à l'analyse ", la loi est "acte d'immédiateté humaine et est humainement immédiat ce dont l'existence enferme la manifestation visible des formations transcendantes ${ }^{41} »$. La sculpture africaine s'avérait donc après coup l'incarnation sensible d'une telle loi : souverainement arbitraire, parce qu'exprimant la transcendance, inanalysable, immédiate dans sa pure visibilité. Nous l'avons dit : pour Einstein, la solution politique devait impliquer une tentative d'appropriation par le sujet moderne de la force métaphysique de la religion dans ce que cette dernière avait de tragique et d'incommensurable. Rien d'étonnant donc à ce que le chapitre "Religion et art africain » explique la présence au sein de la sculpture africaine de toutes ces qualités que, sous l'influence de Sorel, le révisionnisme marxiste d'Einstein attribuait au primitif en tant qu'identité éminemment politique, ce durant les années 1912-1914. Le régime démocratique - majoritaire, discoureur et re-présentatif - se dessinait en négatif de plus en plus obsessivement au fil de ces pages vouées à l'éloge de la distance et de la souveraineté africaines : «L'œuvre d'art européenne est devenue justement la métaphore de l'effet, qui incite le spectateur à une liberté indolente. L'œuvre d'art religieuse est catégorique ", affirmait de manière apodictique Einstein ${ }^{42}$.

Forte de son étrangeté culturelle et, par-là même, parfaitement «inadaptée ", la sculpture africaine était capable de déchirer la belle apparence du présent. Elle obtenait ainsi, malgré elle, une fonction politique inattendue ${ }^{43}$. Elle devenait la preuve contrastante et incongrue - la preuve "anachronique " dirait Didi-Huberman - qu'une sourde catastrophe se déroulait, sans répit, au sein de la démocratie moderne - ce régime des lois équivoques, du consensus et des discussions sans fin. Par sa forme catégorique et concentrée, la sculpture africaine venait couper court au cauchemar de l'histoire, au cortège du nouveau qui n'était au fond que le retour du même. La sculpture africaine devenait ainsi pour Einstein une manifestation concrète surprenante de l'image motrice de Sorel. Mais si cette dernière était mentale, l'image motrice d'Einstein était déjà là et elle pouvait agir de manière fulgurante. Et si Sorel attribuait, cyniquement, à son image motrice un caractère infini par le fait qu'elle n'était pas censée se réaliser en tant que telle dans l'histoire, Einstein a beaucoup cru dans sa vie à l'art comme une prophétie autoréalisatrice, même si cette réalisation n'était jamais définitive ${ }^{44}$. Doté d'une double nature, faite de matière et d'esprit, l'art moderne s'est souvent prévalu d'être le lieu par excellence du « déjà ». Depuis la religion romantique de l'art, les artistes ont opéré leur propre sécularisation du salut en s'appropriant le dogme de l'incarnation christique : ils pouvaient rendre sensible « déjà » ce qui n'était "pas encore » réalisé au sein de l'histoire ${ }^{45}$. Einstein s'inscrit très exactement dans cette tradition messianique de l'art. Mais son impatience apocalyptique déplaçait l'accent du temps sur le «maintenant » de l'œuvre d'art. Ce que le néoclassicisme, soucieux de chasser de la sculpture le temps corrupteur de la nature, appelait «l'instant fertile» se transfigurait chez Einstein, en manque de religion, en «Jugement», temps éclaté, révolution subjective.

Mais il faut s'arrêter un peu plus longuement sur le choix d'Einstein de placer l'instant révolutionnaire dans la sculpture africaine, car ce choix implique un renversement logique, temporel et idéologique capital. En effet, c'est dans les pages de Negerplastik, mais lui-même n'en était peut-être pas encore conscient, qu'Einstein recourt pour la toute première fois à un mécanisme logique qui allait

\section{- $\bullet$}

41. Id., «Das Gesetz » (publié en 1914 dans Die Aktion ], Werke, t. I, op. cit., p. 206.

42. Id., La Sculpture nègre, op. cit., p. 29-31.

43. Le terme d'《adaptation » (Anpassung] allait devenir très important pour Einstein à partir du milieu des années 1920. Il était doté d'une connotation péjorative et signifiait l'assimilation du monde extérieur à l'expérience préexistante du sujet, l'imitation par le présent du passé fossilisé. Inversement, une œuvre d'art, par exemple, qui était «inadaptée » au sujet détenait le pouvoir de le transformer.

44. Einstein pensait-il, à ce moment de sa vie, qu'il pouvait y avoir un Jugement final, que la révolution aurait donc pu offrir une solution définitive, en clôturant ainsi l'histoire? Ou pensait-il plutôt que l'histoire était faite d'une série de Jugements décisifs, mais provisoires, et de leurs échecs? Cet inachèvement constitutif de la révolution apocalyptique serait, à certains égards, plus proche de Sorel; mais aussi plus proche du messianisme juif, qui a pu laisser des traces dans la pensée einsteinienne. Cette question reste ouverte.

45. Sur le «déjà » et le «pas encore » du messianisme chrétien, $c f$. les pages magnifiques écrites par Karl Löwith dans son Histoire et salut (op. cit.), et, à sa suite, par Rudolf Bultmann, Histoire et eschatologie, trad. Roger Brandt, Neuchâtel, Delachaux et Niestlé, 1959. Pour la temporalité du « déjà » et du «pas encore » dans l'art, cf. Éric Michaud, Un art de l'éternité. L'image et le temps du national-socialisme, Paris, Gallimard, 1996 ; id., «Déjà là, mais encore à venir. Le temps de l'homme nouveau en Allemagne, 1918-1945 », in La Fabrique de l'homme nouveau (dir. Jean Clair), Ottawa, musée national des BeauxArts du Canada-Gallimard, 2008, p. 29-35. 
- $\bullet$

46. Cf. Ernst Bloch, Héritage de ce temps, trad. Jean Lacoste, Paris, Payot, 1978.

47. Il s'agit d'une thèse capitale de la pensée de Mondrian. Cf. par exemple son texte «Le néoplasticisme. Principe général de l'équivalence plastique » (1920), in Piet Mondrian, Écrits français, Brigitte Leal (éd.), Paris, Éditions Centre Pompidou, 2010, p. 27 sq.

48. Siegfried Kracauer, L'Histoire des avant-dernières choses, trad. Claude Orsoni, Paris, Stock, 2006. devenir très important pour sa pensée d'après-guerre : nous voulons parler de la conversion du passé au futur et du revirement de la conservation en révolution. En quoi consistait cette double conversion? Dans cette ère centrifuge qu'était la modernité, caractérisée par le "vide » démocratique, l'atomisation subjective et la temporalisation sans fin, les modèles conservateurs de l'histoire détenaient, selon Einstein, le véritable potentiel révolutionnaire. Car s'il abhorrait la dialectique en tant que méthode de la réconciliation démocratique, Einstein l'honorait comme devise d'opposition et de contraste dans sa propre apocalypse politique. Ainsi la sculpture africaine, qui était à strictement parler le produit d'une culture fermement fixée - articulée sur une hiérarchie, sur une tradition et sur des rites, réglée sur un rythme aussi précis que la trajectoire du soleil -, se comportait-elle de manière révolutionnaire dans le contexte relâché, équivoque, individualiste et infiniment prosaïque de la démocratie moderne. Autrement dit, l'efficacité actuelle de la sculpture africaine dépendait de son inactualité même. En ce sens, la révolution préconisée par Einstein a toujours été une révolution à rebours (il devait théoriser cette idée dans les années 1920 et 1930). Dans la modernité, monde plus asynchrone que jamais parce que soumis grâce à la technique et à l'expansionnisme capitaliste à la "contemporanéité du non-contemporain ${ }^{46}$ ", l'Afrique représentait, de manière générale, ce passé que l'Europe avait laissé derrière elle en succombant à la tyrannie de l'humanisme.

Mais aussi, le révisionnisme marxiste d'Einstein était prêt à investir une bonne dose d'espoir sur la forme de la sculpture africaine, parce que cette forme était une traduction exemplaire des lois de vision propres à l'« ontologie africaine ». Dans son contexte historique propre, la sculpture africaine faisait œuvre de conservation en transmettant ces lois à son adorateur présumé. Ces lois visuelles obéissaient à des postulats ontologiques et ces derniers constituaient, bien sûr, le véritable contenu de la contamination révolutionnaire du sujet moderne. Les lois de vision auxquelles se conformait la sculpture africaine transmettaient autant de types de rapports entre l'homme et le monde. Dans le monde africain tel qu'Einstein le voyait à partir de son présent, l'homme n'était pas au centre de la création. Einstein retenait donc d'abord un principe de non-dévoration : le sujet ne s'appropriait pas l'objet en le conformant à sa propre image et à sa propre temporalité, mais négociait au contraire avec l'altérité, que ce soit le dieu ou les animaux, pour établir un équilibre de moi et de non-moi, de vie et de mort. Face à l'imitation humaniste, se dessinait l'alternative de l'identification mimétique de l'homme au monde. Et si la tyrannie du sujet moderne avait même réussi à fondre le bloc sculptural dans le temps, l'inverse se produisait en Afrique, où le devenir humain était régulièrement absorbé dans la stabilité imperturbable de l'être divin. C'est pourquoi aussi la corporéité courbe du sujet (le terme est de Piet Mondrian, qui entendait par là l'autosuffisance matérialiste de l'homme ${ }^{47}$ ) était contredite par le cubique statique de la sculpture ou les tatouages imprimés sur les corps des danseurs en Afrique. Einstein espérait que les artistes modernes qui s'intéressaient à la sculpture africaine essaieraient d'actualiser son attitude ouverte, relationnelle et métamorphique envers le monde. À l'aide des images et de manière d'abord subjective : voilà comment il espérait faire advenir la révolution, qu'aucune maturation des conditions matérielles ne saurait faire surgir de l'histoire.

Einstein nous avait prévenus : toute histoire est interprétée à partir du présent. Selon Siegfried Kracauer, l'« utilité du présent » dans l'écriture de l'histoire était une conséquence du relativisme historiciste ${ }^{48}$. Pour Einstein cependant, certains présents étaient plus universels que d'autres - le sien propre par exemple, pour 


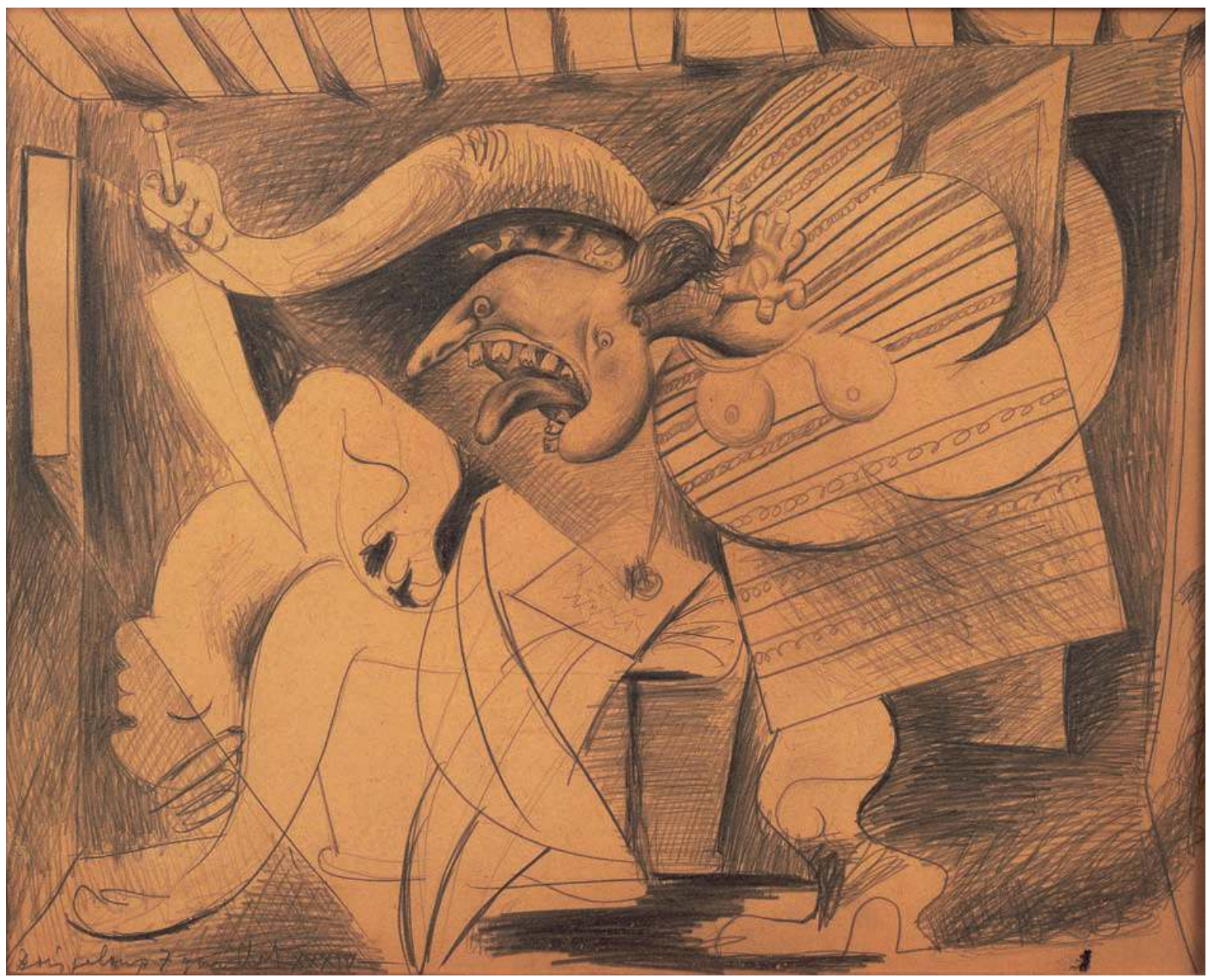

Fig. 9 Pablo Picasso, Le Meurtre. Assassinat de Marat, 1934, Paris, musée Picasso, dist. RMN / Béatrice Hatala ๑ Succession Picasso 2011.

lequel il revendiquait une objectivité qu'il avait refusée aux lectures précédentes du primitif. Cela était dû en partie à l'intention dépréciative et, le plus souvent, raciste de ces lectures vis-à-vis de leur objet. Rejetant les interprétations ethnologiques fondées sur la «valeur d'usage» des artefacts et insistant sur le fait que l'art africain, en raison des migrations, de certaines pratiques guerrières et de son développement stylistique "acausal», était littéralement insaisissable, Einstein retrouvait la terre ferme dans les objets mêmes. Ce que nous refusaient l'histoire et la géographie incertaines de l'Afrique, il le détectait dans les artefacts conservés, car ils étaient immédiatement visibles et saisissables. Il puisait sa croyance en l'objectivité de sa méthode dans les lois de vision qu'il détectait dans la forme de ces objets. Car il ne faisait pas de doute pour Einstein, pas plus que pour Aloïs Riegl ou Heinrich Wölfflin, que «l'analyse des formes [...] demeure dans le champ du donné immédiat, car on n'a que quelques formes communes à présupposer. Celles-ci, cependant, en tant que formes, expriment et les manières de voir et les lois de la vision; elles imposent justement une connaissance qui demeure dans la sphère du donné immédiat 49 . " La boucle est bouclée : les lois de vision sur lesquelles Einstein entendait fonder l'objectivité de son argumentation
-

49. Carl Einstein, La Sculpture nègre, op. cit., p. 21. 
étaient finalement aussi «tautégoriques » - « univoques et impératives » - que le mythe ou la "loi " religieuse imposée au regard par la forme. La méthode einsteinienne se trouvait donc contaminée par son objet; c'était à ce prix qu'Einstein pouvait construire son propre mythe de l'art.

\section{La césure ou l'asynchronie de l'art et de l'histoire}

Les acteurs de l'histoire ont su finalement accélérer l'avènement de la révolution. Cela n'a pas duré, mais cela a eu lieu. Le moyen fut autrement dialectique que celui préconisé par Einstein dans sa lecture volontairement incongrue de la Negerplastik : ce sont les sacrifices inouïs de la Grande Guerre qui ont poussé à bout les armées du front et de l'arrière, en Russie comme en Allemagne. Einstein a participé dans un premier temps aux conseils révolutionnaires qui se sont formés à Bruxelles, et plus tard à Berlin ${ }^{50}$. Quand la révolte spartakiste a éclaté, Einstein n'avait plus de raison d'attribuer un quelconque pouvoir politique à l'art. Ce dernier avait après tout hérité de la malédiction propre à tout médiateur : il restait cloué dans le temps intermédiaire, entre le "déjà " et le "pas encore». Pire : l'art semblait maintenant à Einstein tout simplement en retard. Dans la mesure où l'esprit et la matière, la «forme » et la "force " coïncidaient enfin au sein de l'histoire, il pouvait appeler les Geistigen («les hommes de l'esprit ") à se ranger derrière les masses, qui ont su se mettre en mouvement sans obéir à un quelconque mythe visuel. Et parce que les expériences formelles des artistes modernes n'avaient pas réussi à transgresser leur cadre subjectif, elles basculaient maintenant pour Einstein du côté de la vanité et même de la réaction.

Le texte où il devait parler d'art primitif après Negerplastik était écrit en 1919. Il y déclarait : "L'art européen est pris dans le processus de la différenciation capitaliste. L'époque des fictions formelles touche à sa fin. Avec le déclin de l'économie du continent s'effondre aussi son art. [...] que peut encore apporter l'art exécuté par des petits-bourgeois douteux pour les possédants, quel peut être son apport à une société fonctionnelle? [...] Toute œuvre d'art est une œuvre de snobisme réactionnaire, préhistorique, si elle n'est pas subordonnée à la transformation sociale qui lui confère seule un sens ${ }^{51}$. " Produit de la différenciation capitaliste des facultés individuelles, l'art moderne n'avait pas réussi à devenir "primitif» durant ces années. Et s'il devait suivre le déclin du capitalisme qu'Einstein espérait, c'était parce qu'en tant que produit «surdifférencié ", il était dépourvu de "fonction" dans la nouvelle société qui était en train de se former. L'art moderne était un objet fossile de la préhistoire capitaliste. Sa valeur d'usage d'alors était d'affirmer le sujet "autonome" par le truchement de la vision anthropocentrique et répétitive qu'il procurait : la représentation de l'« objet », toujours identique à lui-même, ravitaillait la possession capitaliste au point de faire croire qu'elle relevait d'un droit naturel. Quant à l'art moderne, il constituait désormais aux yeux d'Einstein le produit le plus abouti de la surdifférenciation capitaliste; il était ultramoderne et, par-là même, ultraréactionnaire : "Cet art livre au bourgeois la fiction d'une révolte esthétisante qui induit l'abréaction "psychique" inoffensive de tout désir de changement. " On le voit : l'art ne réussissait pas à sortir de son statut re-présentatif. Ses expériences les plus téméraires restaient des re-présentations de la révolte, servant même de défouloir. Le renversement causal qu'opérait ce petit texte qu'était «Sur l'art

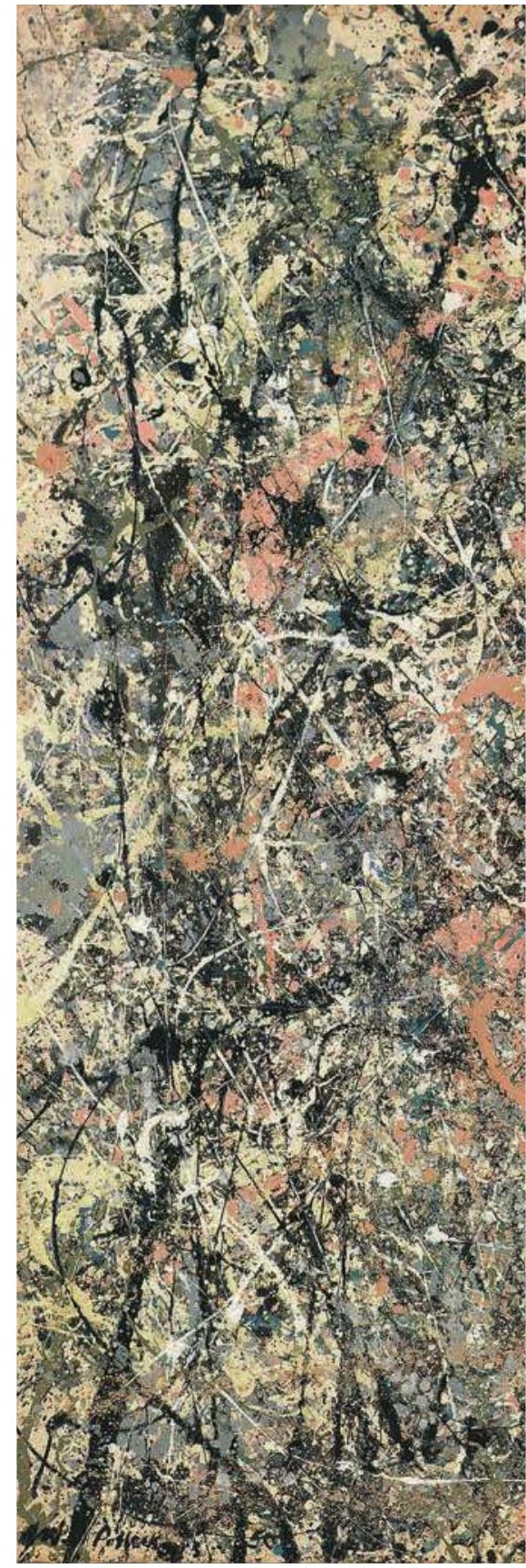

-

50. Cf. Charles Haxthausen, «Bloody Serious: Two Texts by Carl Einstein », October 105, 2003, p. $105-118$

51. Cf. la traduction de ce texte dans ce numéro, intitulé «Sur l'art primitif », p. 185. 


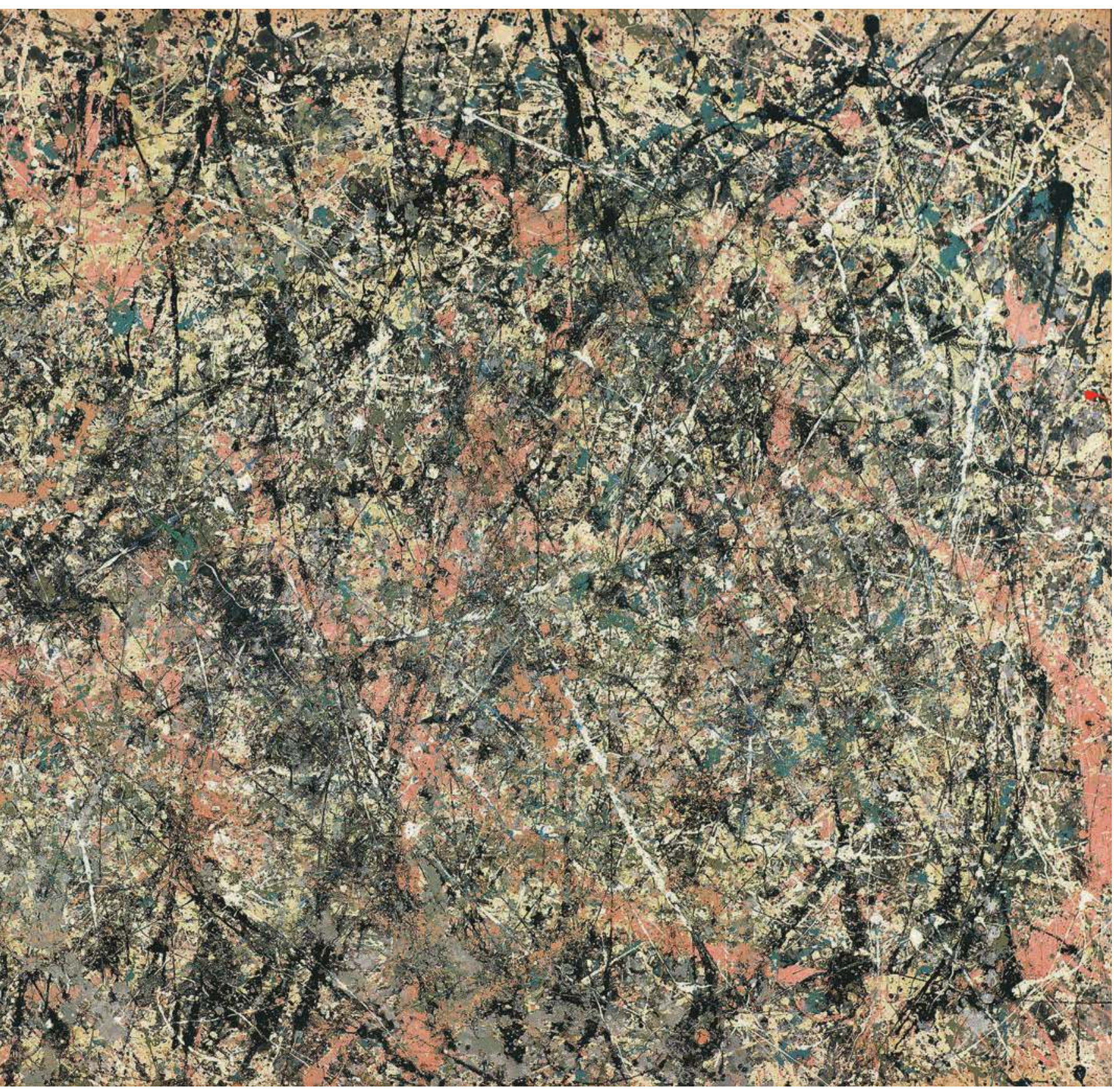

Fig. 10 Jackson Pollock, Lavender Mist: Number 1, 1950, The National Gallery of Art, Washington DC / The Bridgeman Art Library @ ADAGP, Paris 2011. 


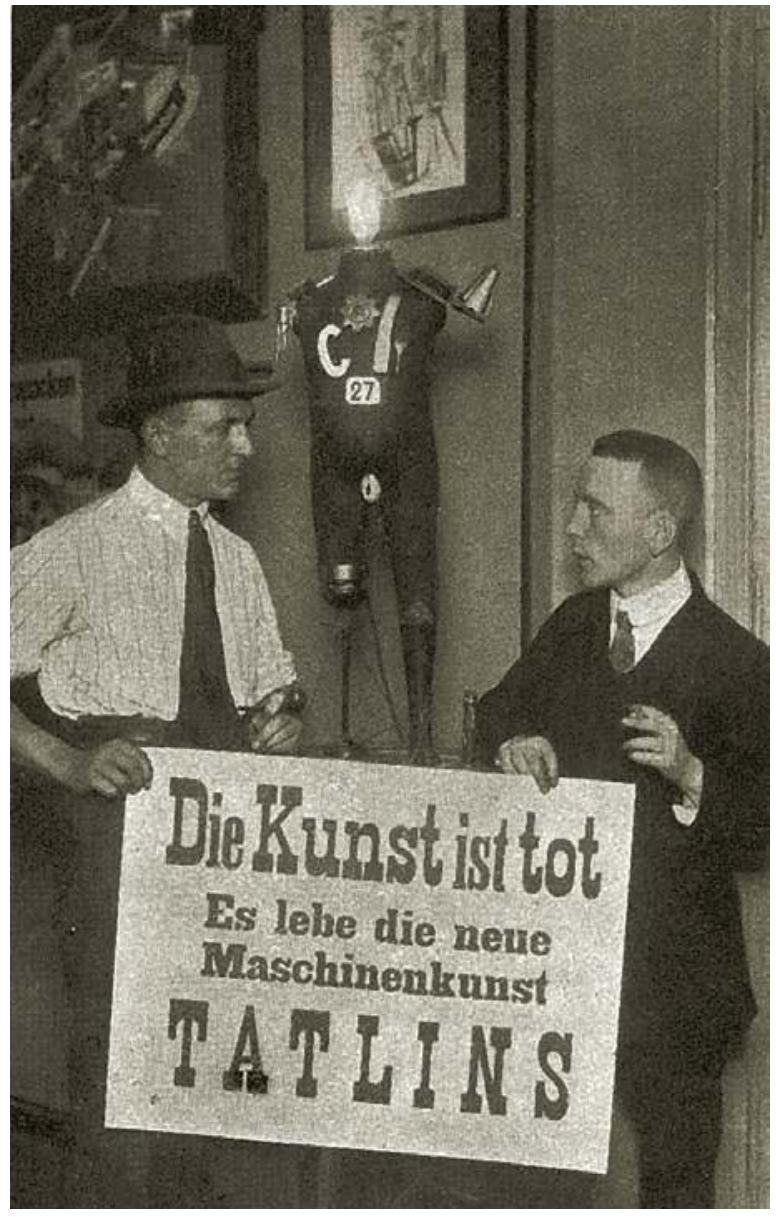

primitif " par rapport à Negerplastik était donc le suivant: dépourvue de possessions, libre de tradition esthétique et, croyait-il, du trop-plein de l'histoire, c'était à la masse d'engendrer l'art qui lui convenait et non l'inverse.

Ces thèses peuvent expliquer pourquoi Einstein a participé, au même moment, à quelques activités dadaïstes, notamment en tant qu'éditeur d'écrits politiques. Proche de la branche marxiste du groupe plutôt que de sa branche anarchiste, il avait travaillé avec l'éditeur de Malik Verlag, Wieland Herzfelde, et les deux photomonteurs George Grosz et John Heartfield. Dans son texte "Sur l'art primitif ", Einstein adoptait une position semblable à celle des deux artistes dadaïstes lorsqu'ils posaient devant la caméra en tenant un panneau sur lequel ils avaient écrit : «L'art est mort. Vive l'art de la machine de Tatline. » L'art dont ils célébraient la fin était celui-là même qu'Einstein avait fustigé dans sa Negerplastik : l'art qui permettait au sujet bourgeois de préserver son autonomie dévorante. Mais, comme Einstein, les deux dadaïstes considéraient aussi que les expériences les plus récentes de l'art moderne n'avaient pas réussi à désobéir à cette finalité. Bien au contraire, les -ismes constituaient le sommet de l'accumulation surdifférenciée de l'histoire de l'art, parce qu'ils exigeaient la connaissance du passé qu'ils contredisaient. Par-là même, les expériences modernistes restaient muettes face aux masses "primitives", dépourvues de patrimoine esthétique ou autre. C'était sans doute cette convergence de vues entre les deux dadaïstes et Einstein au sujet de l'art moderne qui

Fig. 11 George Grosz et John Heartfield à la Dada Messe, Berlin, juin 1920. Photographie anonyme.
- -

52. Sur les rapports entre Einstein et Dada à Berlin après la révolution spartakiste, cf. Uwe Fleckner, « The Real Demolished by Trenchant Objectivity: Carl Einstein and the Critical World-View of Dada Verism », in The Dada seminars, Leah Dickerman et Matthew S. Witkovsky (éd.), Washington, Center for Advanced Study in the Visual Arts, 2005, p. 57-82; Charles Haxthausen, «Bloody Serious: Two Texts by Carl Einstein », October, art. cit. ; Maria Stavrinaki, «Dada inhumain : le sujet et son milieu », Les Cahiers du musée national d'Art moderne 103, 2008, p. 66-99. était célébrée, non sans une bonne dose de malice de la part des deux artistes, dans un "papier collé » détourné qu'ils lui dédièrent. À la reproduction de Tête de jeune fille (1913) de Picasso, ils intégraient la photographie d'un soldat. Le titre qu'ils donnaient à cette œuvre d'art "corrigée » (catégorie expressément conçue pour la Première Foire internationale dada en 1920) était Pablo Picasso : la vie heureuse (Dédié au Dr Karl Einstein). Ce n'étaient pas les lois visuelles qui pouvaient changer l'histoire, mais l'inverse. Quand le document photographique faisait son entrée critique dans l'espace formaliste du cubisme, la «vie heureuse " procurée par l'art "abréactif» était brusquement arrêtée au profit de la vraie vie ${ }^{52}$.

\section{Retour des ancêtres, retour à l'art}

Mais, comme nous le savons, la révolution n'a pas abouti en Allemagne et elle s'est même très mal terminée pour les communistes. La "catastrophe" quotidienne a repris de plus belle quand la social-démocratie a élargi son pacte en incluant l'extrême droite à ses alliés démocratiques habituels. Puis les choses ont repris leur cours, mais à l'ancien Empire s'était substituée entretemps une toute jeune République. Peu de temps après, Einstein devait trouver à nouveau dans l'art la promesse de la catastrophe violente de l'histoire. Désormais, la révolution n'était plus conçue uniquement comme la rupture apocalyptique du temps, mais aussi comme l'apport impromptu d'une mémoire ancestrale involontaire qui s'empa- 
rait du sujet, et en particulier de l'artiste. Cette mémoire était dotée d'un potentiel révolutionnaire qu'Einstein tenait à opposer aussi bien au sac du passé, opéré massivement par le retour à l'ordre, qu'à l'inflation de la mémoire chez un Giorgio De Chirico désabusé. Ainsi, comme par un curieux procédé de récapitulation, Einstein allait répéter dans sa propre vie un schéma que la pensée européenne a mis plus d'un siècle à élaborer : ce qu'il a d'abord cherché loin dans l'espace, dans l'Afrique donc (fût-elle imaginée), il l'a découvert plus tard au fond de lui-même, dans la mémoire collective gravée dans son inconscient ${ }^{53}$. C'était cette mémoire des ancêtres, vouée à des métamorphoses infinies, qui médiatiserait désormais le mythe objectif faisant défaut aux modernes.

Université de Paris I/Centre de recherches interdisciplinaires sur l'Allemagne (CRIA)

Maria.Stavrinaki@univ-parisı.fr
- $\bullet$

53. Cf. Hayden White, «The Forms of Wildness: Archaeology of an Idea », in Tropics of Discourse: Essays in Cultural Criticism, Baltimore, Johns Hopkins University Press, 1978, p. 150-182.

mots clés / keywords : temporalités // temporalities - primitivisme // primitivism • révolution // revolution $\cdot$ mythe // myth $\cdot$ avant-gardes // avant-gardes.

\section{Résumé / Abstract}

Maria Stavrinaki, Apocalypse primitive. Une lecture politique de Negerplastik - Quels étaient les régimes politiques et temporels de Negerplastik? Démocratie parlementaire et évolutionnisme volaient en éclats par l'impact sensible des sculptures africaines, arrachées à leur histoire. Leur autonomie formelle s'opposait aux foules démocratiques et leur identité spatiale, resserrée dans le présent, contrastait avec la temporalisation de la sculpture moderne et de l'histoire elle-même. Le temps de l'art démagogue, de la répétition propre au capitalisme et à l'historicisme, de l'attentisme socialiste aussi était brisé. L'idée sorélienne de la motricité du mythe social se transposait dans les artefacts africains, incarnations paradoxales de la révolution. L'emprise de l'art sur l'histoire s'effectuait pour Einstein par les suggestions inscrites dans sa nature sensible. De la légalité universelle de la vision humaine, il déduisait l'efficacité politique de l'art moderne attentif à l'art «primitif ». La sculpture africaine changeait ainsi de régime ontologique et temporel : d'instrument conservateur, elle se transformait en outil révolutionnaire. Mais une aporie irrésolue, une asynchronie radicale, déterminait le rapport entre l'art et la politique chez Einstein.
Maria Stavrinaki, Primitive Apocalypse. A political reading of Negerplastik- Of what kind were the political and temporal regimes in Negerplastik? Parliamentary democracy and evolutionism were shattered by the sensory impact of african sculptures, torn from their own history. Their formal autonomy was opposed to democratic crowds and their spatial identity, tightened within the present, was in contrast with the temporalisation of modern sculpture and of history itself. The time of demagogic art, of repetition characteristic of capitalism and historicism, but also the one of socialist expectations was broken. The sorelian idea of social myth's motricity was transposed to african artefacts, these paradoxical incarnations of the revolution. The influence of art on history was taking place according to Einstein through the suggestions inherent in its sensory nature. From the universal legality of human vision he deduced the political efficiency of modern art attentive to "primitive" art. African sculpture was thus changing ontological and temporal regime: from a conservative instrument it turned into a revolutionary one. And yet an unresolved aporia, a radical asyncrhonicity, determined the relation between art and politics in Einstein's thought. 